\title{
Viscous Fingering During Heavy-Oil Displacement by Chemical Solutions: Emulsion Instability and Partial-Miscibility
}

\author{
Jungin Lee ${ }^{1}$, Jingjing Huang ${ }^{1}$, and Tayfun Babadagli ${ }^{1}$ \\ ${ }^{1}$ University of Alberta
}

September 2, 2020

\begin{abstract}
The viscous fingering morphologies at a particular stage of emulsion displacements are observed to follow similar development patterns as that of the partially-miscible viscous fingering behavior (existent between the commonly studied fully miscible and immiscible systems). Chemically-induced viscous fingering before and after the finger droplet development resembling the partially-miscible fluid behavior is investigated using fractal dimensions and various -classic and modified- dimensionless scaling groups. We demonstrate, through the quantitative analysis using the empirical data collected from our previous experimental study, that the finger droplet formation morphologies observed in partially-miscible injection are indicative of an emulsion hydrodynamic instability behavior. The dominant parameters responsible for such droplet formation and their relationship to fractal dimension and hydrodynamic stability are mapped in a phase diagram with the original experimental images.
\end{abstract}

\section{Introduction}

Since Saffman and Taylor's (1958) pioneering study, it has become a well-established notion that when a low viscosity fluid displaces a high viscosity fluid, viscous fingering results owing to fluid mechanical instability. Even though there is a lack of consensus on the mechanisms that determine the viscous fingering behavior at reservoir conditions (Settari et al. 1977), it is an effective mechanism that dominates the displacement efficiency; this domination might be more critical with complex fluids such as emulsions, colloids, foam, and reactive chemicals than with simpler structures (fluid pairs) causing purely immiscible and miscible interaction. Therefore, studying viscous fingering is essential in developing a deeper insight into the complex multiphase flow behavior in porous media (Jackson et al. 2017).

Traditionally, fully miscible or immiscible fluid systems are employed to observe these fingering pattern morphologies to understand the physical phenomena of multi-phase flow (Fu et al. 2017). However, in that process, a large quantity of fluid systems in the gap (Figure 1 ) between these two extreme cases of immiscibility (Chuoke et al. 1959; Chen 1989; Ferer et al. 2004; Naderi and Babadagli 2011) and miscibility (Nittmann et al. 1985; Yortsos 1988; Tan and Homsy 1992; Gharbi et al. 2001; Nagatsu et al. 2007; Bischofberger et al. 2014; Chui et al. 2015) was not appropriately addressed.

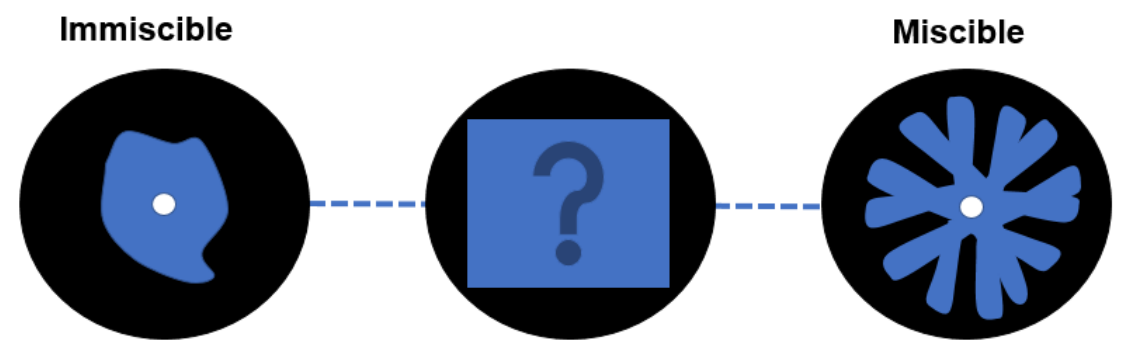


Figure 1-The gap between immiscible viscous fingering and miscible viscous fingering.

Suzuki et al. (2016) noted in their study with sodium sulfate and polyethylene glycol that the phase separation via spinodal decomposition outside the stage of metastability is associated with multiple droplet formation (due to the thermodynamic instability as opposed to hydrodynamic instability). Following their study, Fu et al. (2017) reported in their study of gas-in-oil injection for enhanced oil recovery at highpressure conditions, the transition of the fluid system from immiscible to partially-miscible results in higher occurrences of droplet formation than finger formations.

This leads us to a question regarding one of the rarely studied but commonly encountered case: What about emulsions ? Emulsions are defined as fluid systems that consist of the oleic phase and aqueous phase and can achieve mixing with the addition of surface-active agents. Even without the addition of chemicals, emulsification is a commonly encountered part of crude oil production (Lee et al. 2019; Bruns and Babadagli 2020; Wang et al. 2020) - especially in the case of bitumen as bitumen contains a higher level of indigenous surface-active components such as asphaltene, naphthenic acid, humic acid, etc. relative to the light crude.

In our recent study (Lee et al. 2020), we have provided an extensive set of visual images captured from emulsion visualization experiments, where a heavy oil-saturated Hele-Shaw model was injected with chemicals of various components and rheological properties - which allows us to categorize and capture the universal fundamentals of the chemically induced viscous fingering development. The compact view of the original experimental images of selected samples is available in Figure $\mathbf{2}$ which displays the viscous finger development with chemical addition including AS (alcohol propoxy sulfate), $\mathrm{CTAB}, \mathrm{SiO}_{2}$, and $\mathrm{XG}$ (Xanthan gum). The classification methods for the finger morphologies are elaborated in the cited study.

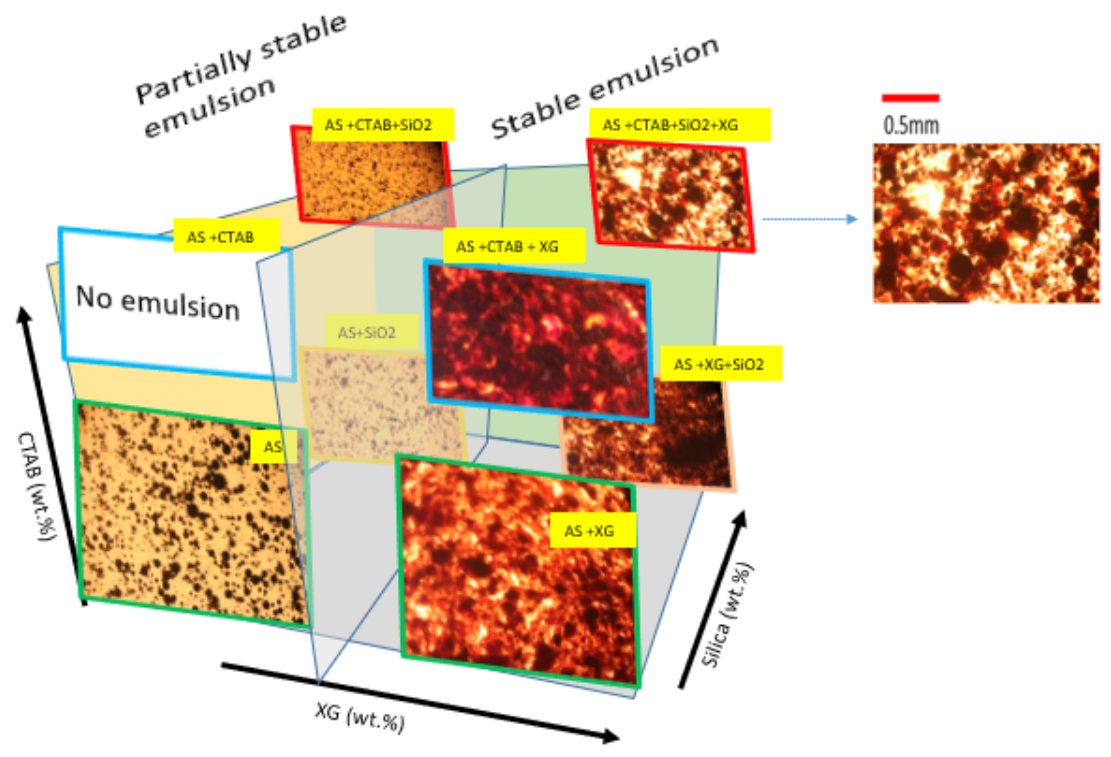




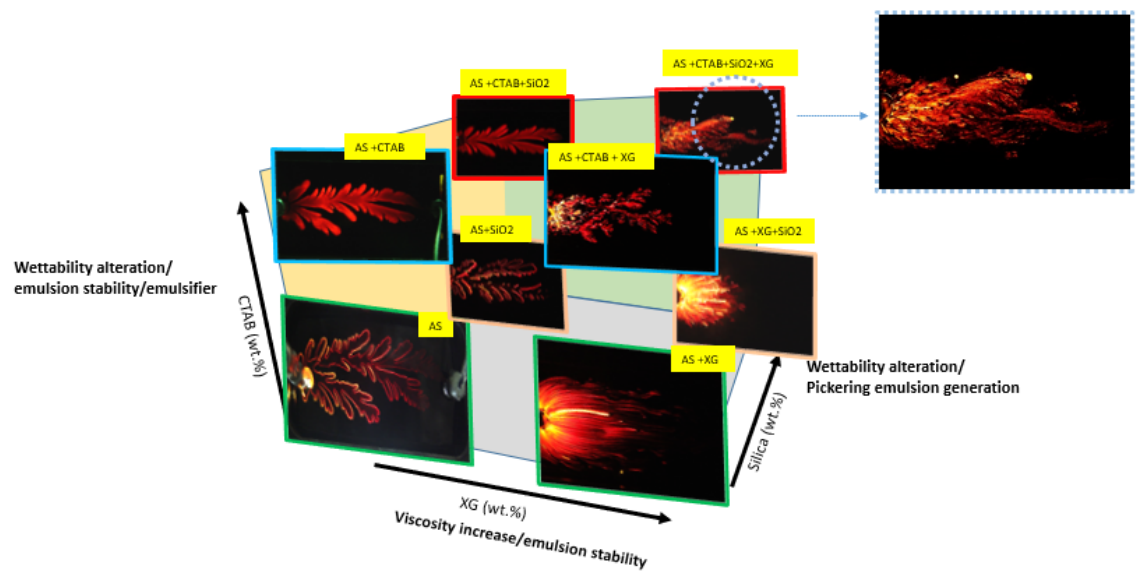

(b)

Figure 2--Summary of viscous fingering experiments (based on Lee et al. 2020) (a): HeleShaw images (b): Micrographs of produced samples *XG: Xanthan Gum, AS: Anionic surfactant.

In our previous visual analysis work using steam and polysorbate at thermal conditions at $200^{\circ} \mathrm{C}$ (Bruns and Babadagli 2020) and cationic surfactant at non-thermal conditions (Lee et al. 2020), droplet generation could be seen in both cases (following the predicted tip-splitting fingers) bearing a striking resemblance in their appearance to the droplet development encountered in partially-miscible fluid flow (Figure 3 ).

\section{(a) Thermal (Pure steam)}
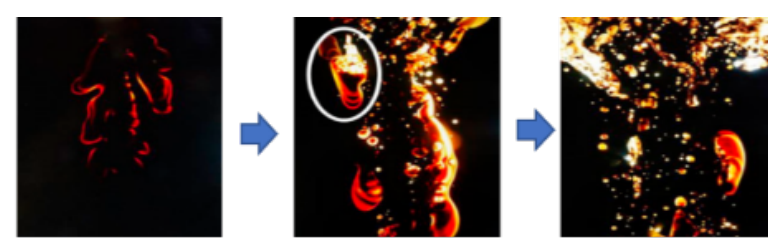

(b) Thermal
(With Tween 80)
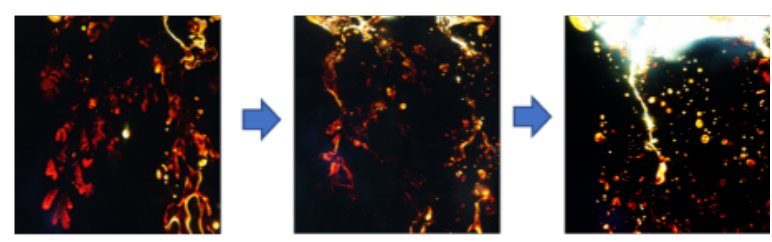

(c) Non-thermal
(with $C T A B$ )
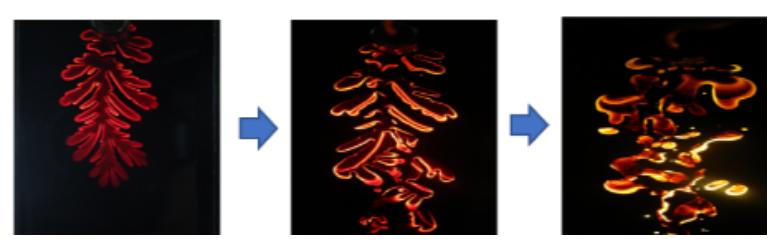

Figure 3-Transitions of the viscous finger and droplet formation (a): steam injection into heavy oil at $200^{\circ} \mathrm{C}$ (b): steam injection with Tween 80 (polysorbate 80 ) into heavy oil $200^{\circ} \mathrm{C}$, (c): CTAB (cationic surfactant) injection at $25^{\circ} \mathrm{C}$.

To investigate further on the droplet development behavior, in this paper, we attempted to account for the observed experimental data with a specified focus on the droplet generation stage of the emulsion flow by comparing the self-similarity behavior of fingers before and after the finger stability loss. To achieve this, 
we first employed a fractal analysis approach. We have specifically selected the solution samples that have resulted in droplet generation at a certain stage of the experiments and the solutions range widely in IFT $(0.02$ to $17 \mathrm{mN} / \mathrm{m})$ and viscosity ratio $\left(7.22 \times 10^{-5} 5.71 \times 10^{-2}\right)$ which makes it appropriate to explore the universal fractal characteristics of in-situ emulsion development in a Hele-Shaw model. Then we investigated the functional relationship between the selected parameters and various dimensionless scaling groups to enhance our understanding of the influence that each parameter has on droplet formation in emulsion flow and explore the relevant mechanisms.

\section{Visualization Experiments}

Visual studies (Hele-Shaw models) are limited to 2D models due to difficulties in obtaining detailed images using 3D models (Fang and Babadagli 2016a-b-c) applying 2D analysis can provide the information necessary for understanding the fundamentals of multiphase fluid flow (Maxworthy 1986; Arnéodo et al. 1989; Hamida and Babadagli 2007; Al-Bahlani and Babadagli 2012; Jha et al. 2013; Fu et al. 2017; Lee et al. 2019). In order to develop insights into understanding the analogy between emulsion instability associated fingering and partial miscibility induced fingering instabilities, we applied a two-dimensional Hele-Shaw model derived from a large quantity of literature available on Hele-Shaw flows for miscible and immiscible flooding. Chemical combinations selected from the screening include AS: Anionic Surfactant, CS: Cationic Surfactant, NF: NanoFluid, and P: Polymer (Table 1 ). Their chemical types are CS: CTAB, NF: Silica, AS: Alcohol Propoxy Sulfate, and P: Xanthan gum. Their associated information (such as active matter, commercial name) along with the detailed experimental set up can be found in Lee et al. (2020). A constant flow rate of $0.1 \mathrm{ml} / \mathrm{min}$ was selected for each injection case. Chemical combinations were selected based on their ability to generate droplets during the experiment.

Table 1-Visualization experiments.

\begin{tabular}{lllll}
\hline Experiment number & Oil viscosity $(\mathrm{cP})$ & Brine type & $\begin{array}{l}\text { Chemicals }(1 \mathrm{wt} . \% \\
\text { each })\end{array}$ & $\begin{array}{l}\text { Chemical viscosity } \\
(\mathrm{cP})\end{array}$ \\
\hline I & $13,850 \mathrm{cP}$ & $\mathrm{NaCl} 10,000 \mathrm{ppm}$, & $\mathrm{CS}$ & 1 \\
& & $\mathrm{CaCl2} 1,000 \mathrm{ppm}$, & & \\
II & $\mathrm{MgCl} 2,100 \mathrm{ppm}$ & $\mathrm{CS}+\mathrm{NF}$ & 1 \\
III & & $\mathrm{AS}+\mathrm{NF}$ & 1 \\
IV & & $\mathrm{CS}+\mathrm{AS}$ & 1 \\
V & & $\mathrm{CS}+\mathrm{P}$ & 494 \\
VI & & $\mathrm{CS}+\mathrm{P}+\mathrm{NF}$ & 791 \\
\hline
\end{tabular}

\section{Fractal Analysis}

Changes in fingering pattern morphology are closely related to fractal behavior (Nittmann et al. 1985; Kawaguchi et al. 2004). Chen (1989) and May and Maher (1989) noted that repeated tip-splitting is associated with higher fractal dimensions of the fingers. Building on the previous observations, comparative analysis between the fractal dimension $(D)$ generated based on the statistical theories from Sharma et al. $(2012)$ and Ferer et al. (1993) and fractal dimension $\left(D_{B}\right)$ from the box-counting analysis using ImageJ (Abramoff et al. 2004) was conducted. The main objective of this particular analysis is to characterize the displacement model before and after the finger stability loss in order to better predict the change in displacement pattern caused by the hydrodynamic instability of fingers.

The results demonstrated that while $D_{B}$ falls in the range of $[1.47 \sim 1.6], D$ is observed to be in the range of $\left[1.3^{\sim} 5.6\right]$, which is notably different in the case of CTAB (experiment I) and CTAB +XG (experiment V) as seen in Figure 4 . Two distinct reasons could be responsible for such pronounced discrepancy: (1) considering that $\log (l)$ was plotted before the fingering instability, the time to reach the normalized finger break time defined as $t B / t T$ for these two experiments is significantly shorter than that of other 
cases (this point will be further elaborated in the next section), and (2) while $t B / t T$ is relatively short for the experiment I and V, finger development occurred at small-scale for both cases (due to reasons such as hydrodynamic stability and/or delayed chemical interaction), and thereby limiting the application of the method for accuracy. Therefore, the fractal pattern scaling terminology (Equation 18a and 18b) from Ferer et al. (1993) and Sharma et al. (2012) could not be strictly applied for the saturation models of Experiment I and Experiment V.

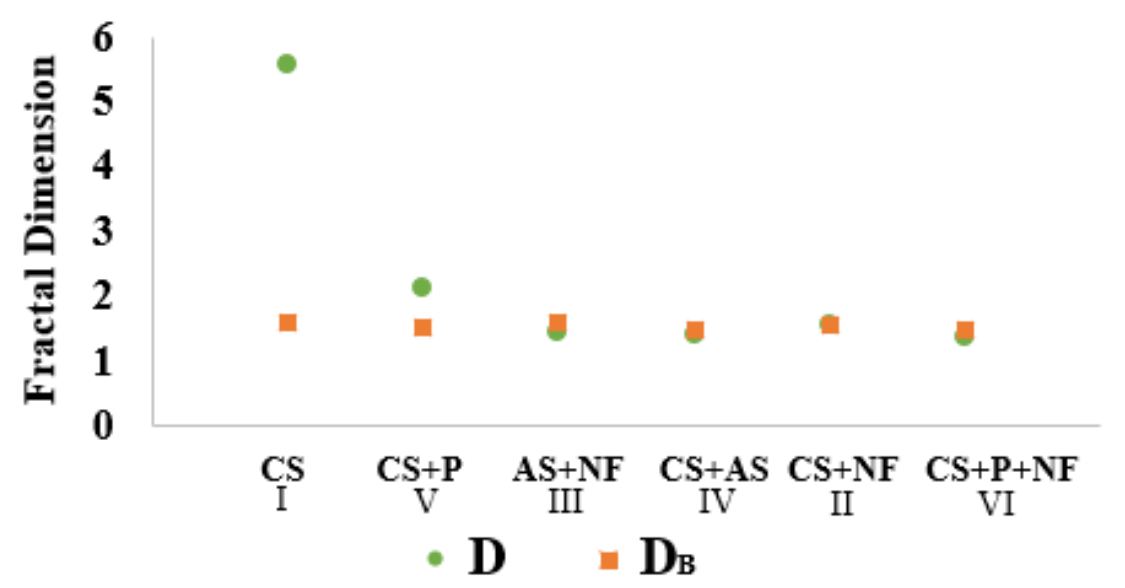

Figure $4-\mathrm{D}$ vs. $\mathrm{D}_{\mathrm{B}}$.

As Figure 4 demonstrates, the fractal dimensions of two different methodologies were observed to be consistent for the rest of the experiment sets despite the dissimilarity in structural and external conditions found in our experiments and that of the previous literature. In order to investigate the effect of fractal numbers $\left(D, D_{B}\right)$ on the saturation profiles, we have further adopted the methodology from the immiscible studies of Sharma et al. (2012) and generated four different profiles (Figures 5-11 ) to investigate self-similar behavior of the chemical samples.

Their descriptions are as follows:

Profile I: $\log (l)$ vs. $\log (t)$, where $(l)$ is the maximum distance traveled by the interface at a time $(t)$.

Profile II: Saturation $(x, t)$ vs. Distance, $x(\mathrm{~cm})$ represents the time evolution of the normalized saturation

Profile III: Saturation as a function of wave speed $\left(x / t_{D}\right)$, wheret $t_{D}$ is the dimensionless time based on the chemical injection volume.

Profile IV: Scaled saturation profiles of $t_{D}{ }^{e} s(x, t)$ vs. $\left(x / t_{D}{ }^{1+e}\right)$. For Figures 5 -10 $D$ was used, and to plot Figure 11, $D_{B}$ was used for comparison purposes. 

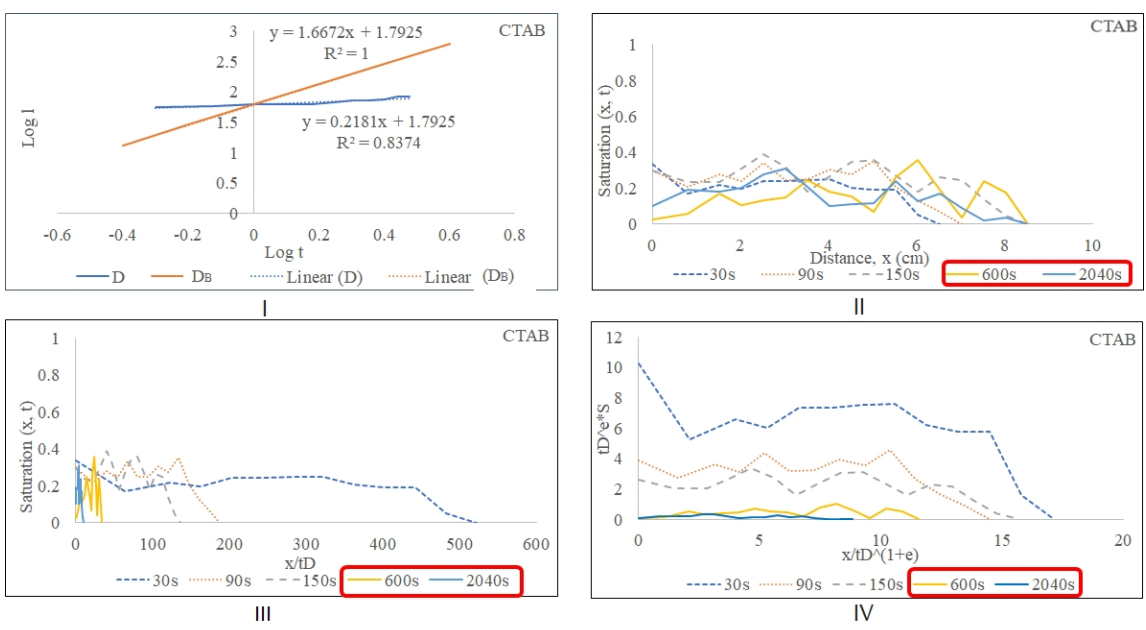

Figure 5-CTAB (Experiment I).
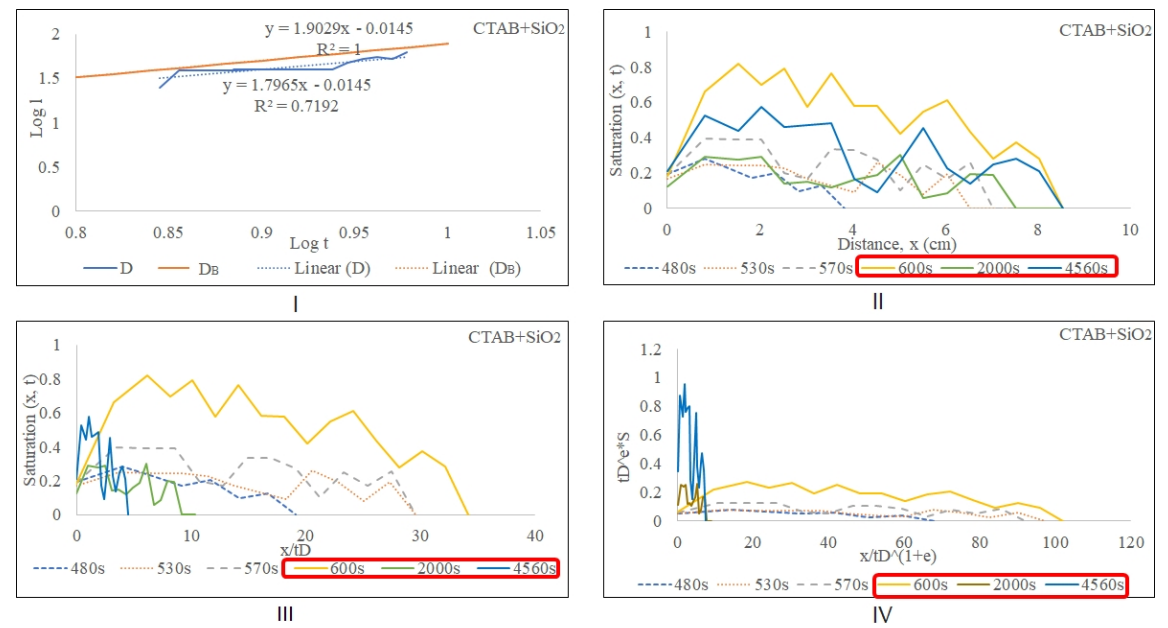

Figure 6-CTAB $+\mathrm{SiO}_{2}$ (Experiment II). 

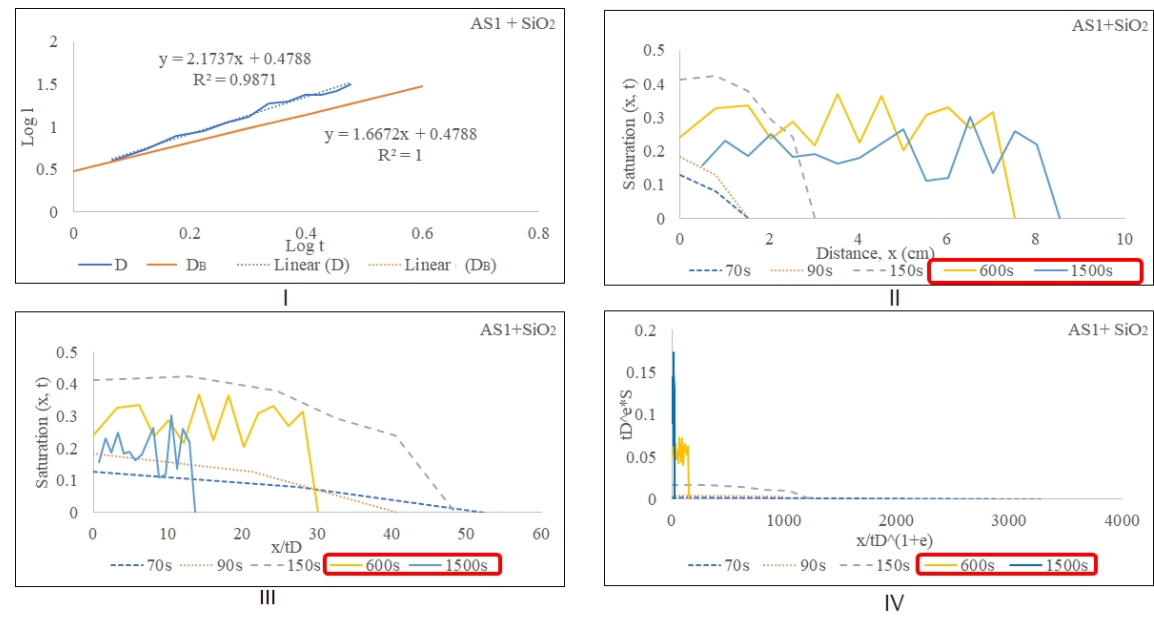

Figure 7-AS1+SiO2 (Experiment III).
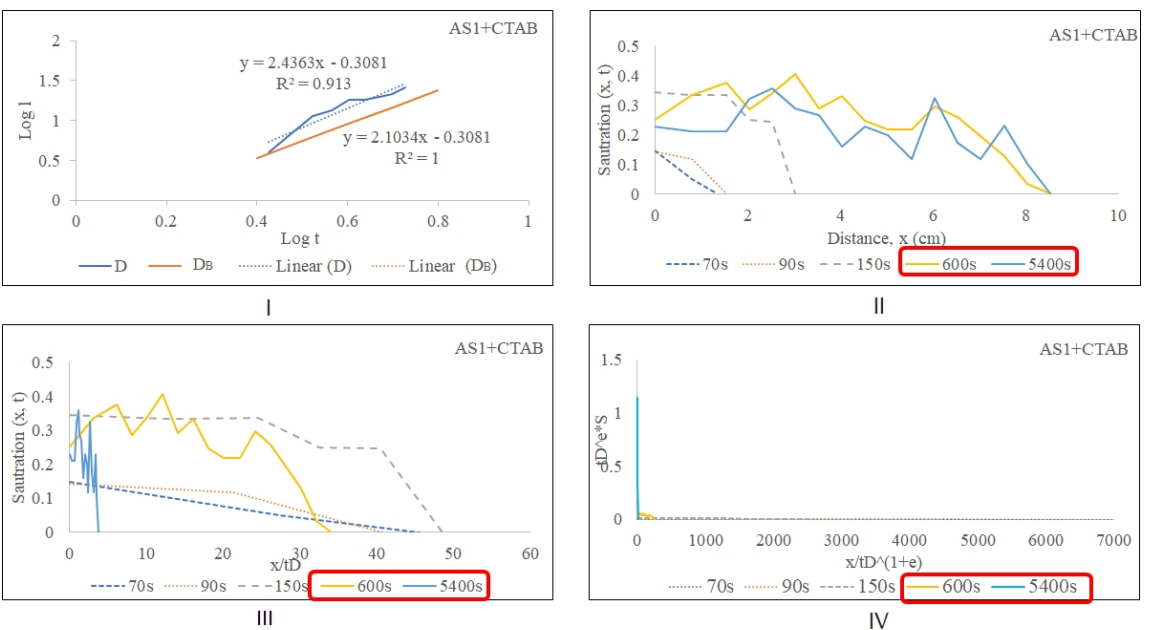

Figure 8-AS1+CTAB (Experiment IV). 

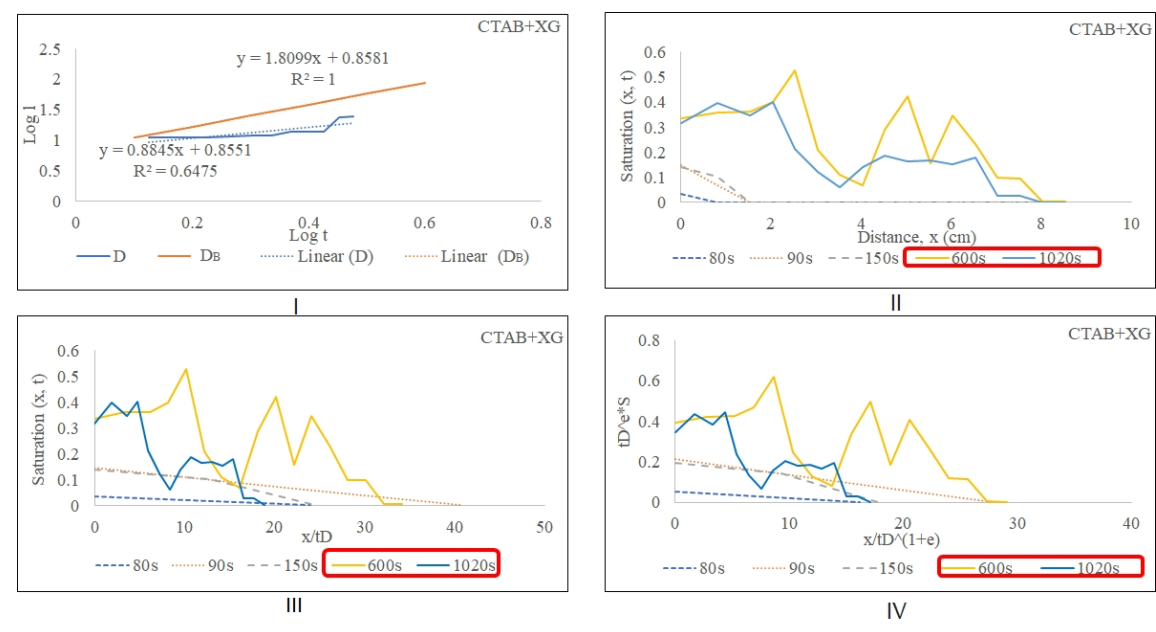

Figure 9-CTAB+XG (Experiment V).
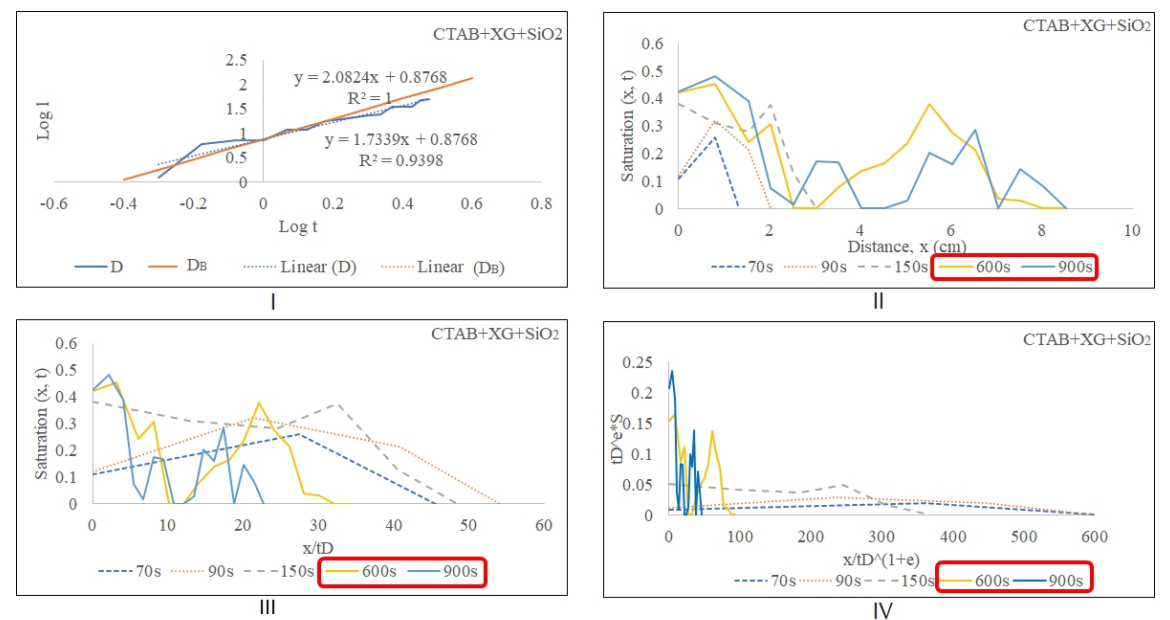

Figure $10-\mathrm{CTAB}+\mathrm{XG}+\mathrm{SiO}_{2}($ Experiment $\mathrm{VI})$. 
CTAB

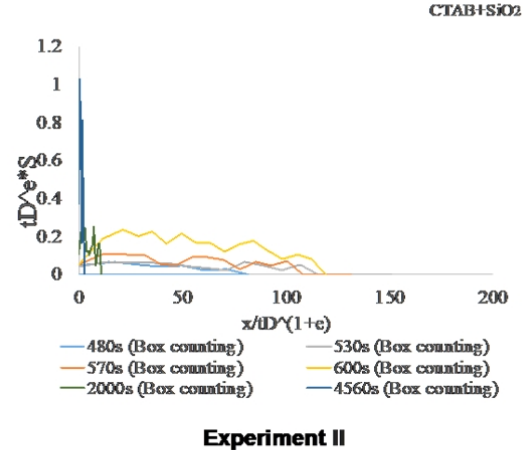

Experiment I
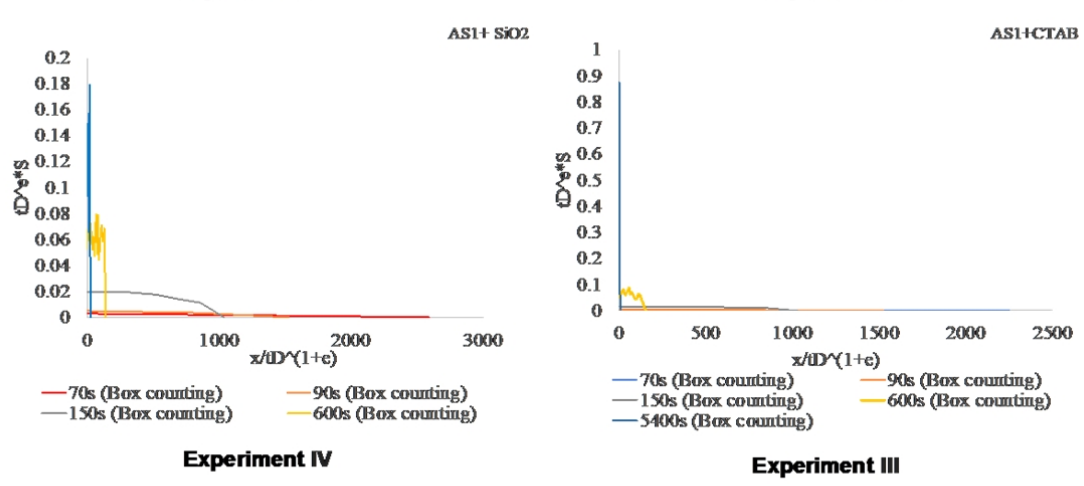

$\mathrm{CTAB}+\mathrm{XG}$
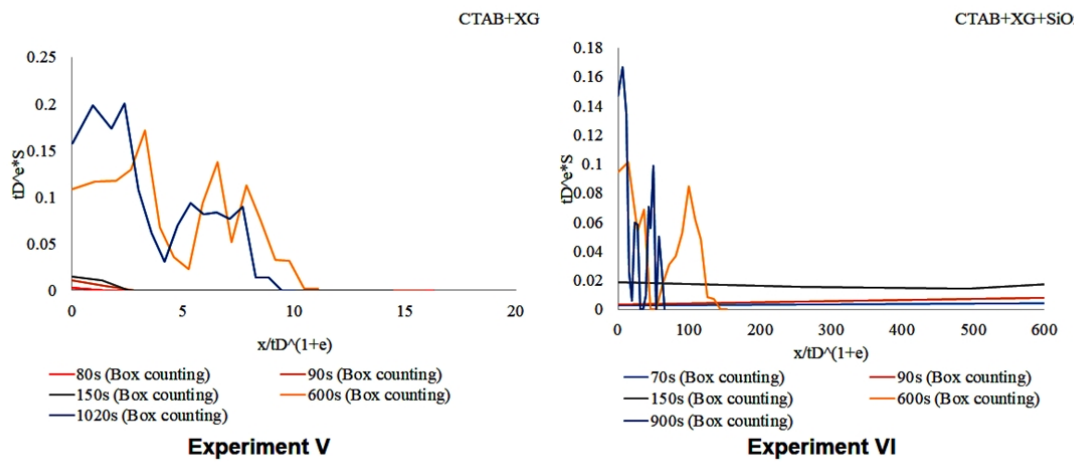

Figure $11-t_{D}{ }^{e} s(x, t)$ vs. $\left(x / t_{D}{ }^{1+e}\right)$ using $D_{B}$.

The saturation profiles are expected not to scale linearly with position and time when using traditional linearflow models. With the collapse of curves when $t_{D}{ }^{e} s(x, t)$ was plotted with $\left(\mathrm{x} / t_{D}{ }^{1+e}\right)$, however, self-similarity is expected to be observed (in accordance with the results from the previous literature). We plotted time vs. saturation for time steps before the finger break and the time steps after the finger break (e.g., $t>600 \mathrm{~s})$. Time steps after the finger break are indicated in a red rectangle in the legend box of Figures 5-11 and their corresponding saturation was recorded. As can be seen, complete collapsed saturation profiles could not be obtained regardless of the adequacy of the range of fractal numbers (for both $D$ and $D_{B}$ ). However, saturation profiles allowed us to observe that there exists a significant difference between the fingering behavior prior to the finger break (which often results in droplet generation - this will be elaborated further in the next section) and after the finger break. And they tend to follow a similar trend of each section; in the earlier stages of experiments, fingers did not reach significant portions of the Hele-Shaw sample resulting in a lack of data points and accordingly smooth curves for most samples. After the finger break, however, significant fluctuations in the graphs can be observed due to the expansion of saturated profiles (in the form of fingers 
or droplets). Therefore, it can be inferred that hydrodynamic instability (in the form of droplet generation) is the determining factor in generating different types of "self-similar" fingering behaviors.

Figure 12 displays the relationship between fractal dimension $(D B)$ and classic capillary number (Equation 1). Since $D$ values for Experiment I and Experiment V were observed to be inordinate, (while the rest were consistent with $D_{B}$ ) , $D_{B}$ were selected for further analysis. As seen, with the capillary number increase, fractal dimensions increase until reaching a peak followed by a relatively slow decrease. Such behavior can be explained by the interplay between surface tension and the viscosity effect. As the surface tension decreases, the fractal number increases (due to the high tip-splitting), however, after the capillary number reaches a certain point $\left(10^{-3} 10^{-3}\right)$, the viscosity becomes the dominant force in the establishment of finger morphology (smoothing the frontal mobility line) which leads to a reduction in fractal number.

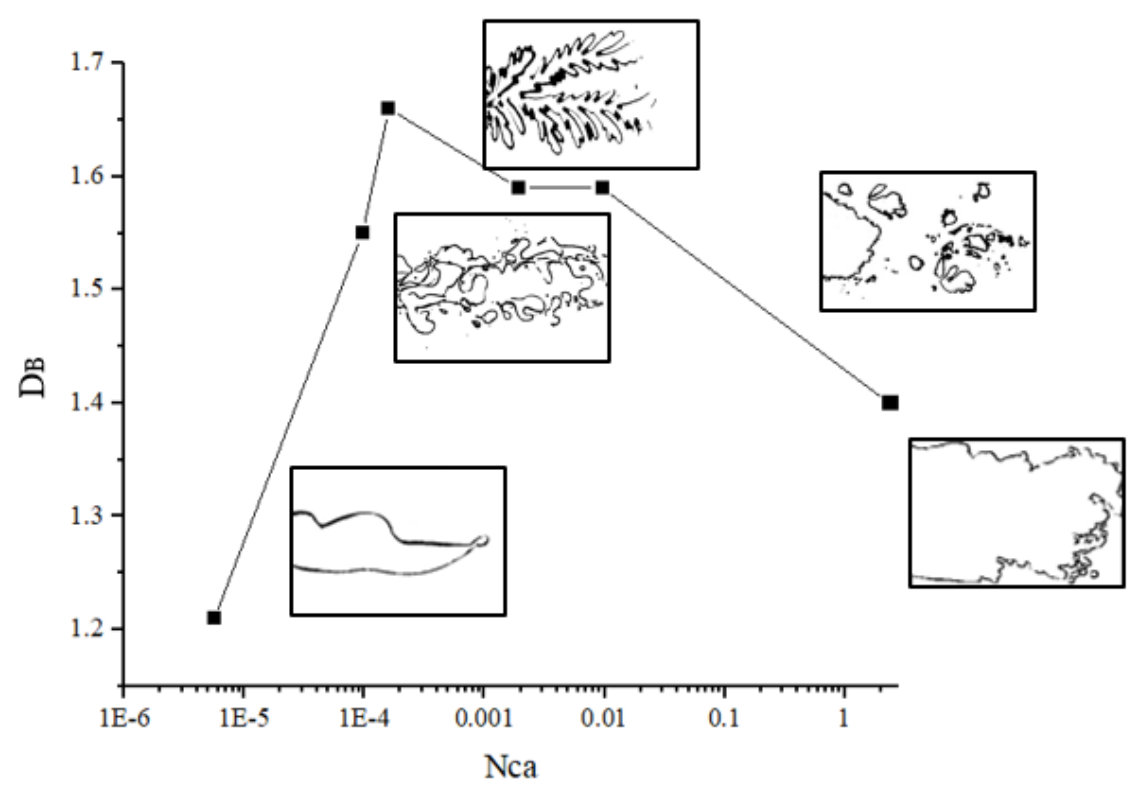

Figure 12-Fractal dimension and capillary number $\left(\mathrm{N}_{\mathrm{ca}}\right.$ in $\log$ scale).

Descriptive parameters and their relationship to dimensionless numbers

To enhance our understanding of the influence that each parameter has on droplet formation in emulsion flow and explore the corresponding mechanism, we have selected dimensionless scaling groups and parameters and investigated the functional relationship between them. The normalized indicators studied include $\frac{L}{L_{y}}$, which is the normalized value of the interfacial length of fingers before the finger break and $L_{y}$ is the vertical length of the Hele-Shaw samples. $N d(b)$ is the number of droplets before the complete finger stability loss. It should be noted that complete finger loss is defined as the loss of an "initiator," equivalent to the equilateral triangle forming an "island" per description by Mandelbrot (1983). $N d(f)$ is the number of droplets at the final stage of the injection. $\frac{t_{B}}{t_{T}}$ is the normalized time to reach finger break, $t_{T}$ is the total time of the injection period. $\frac{t_{P}}{t_{T}}$ is the normalized time for fingers to reach the production port, where $t_{T}$ is the normalized total injectant volume. Both the structural terms and time-dependent terms are considered in the description as functions of chemical parameters as suggested by De Wit (2001). In the past, finger-width was widely suggested as an indicator to determine dynamic surface tension and viscosity in a complex colloidal fluid fingering morphology (Bonn et al. 1995). Association of the chemical reaction with the finger width was also shared by De Wit (2001), Sastry et al. (2001), and Fernandez and Homsy (2003). While the legitimacy of such observations continues to hold, proper finger width analysis does not include droplet presence and therefore, was not considered in this study. The magnitude of the viscous and capillary forces in their 
influence of flow morphologies leading to the fingering loss stage was investigated using (1) classic capillary number $N_{c a},(2)$ modified capillary number $N_{c a}{ }^{*}$, which considers an extra viscosity ratio term $\left(\frac{\mu_{s}}{\mu_{o}}\right)$ to the original capillary number, (3) modified capillary number $\left(N_{c a}{ }^{* *}\right)$, which considers both the contact angle and an extra viscosity ratio term, and (4) viscosity number $(M)$ which considers viscosity ratio and contact angle. Droplet break up behavior dependent on the velocity was also investigated using the (5) Weber number $(W)$ which describes the rate of inertial forces to capillary forces, and (6) modified Weber number which includes the wettability effect.

$N_{c a}=\frac{\mu_{s}}{\sigma}(1)$

$N_{c a}{ }^{*}=\frac{\mu_{s}^{2} \nu}{\sigma} \frac{1}{\mu_{o}}(2)$

$N_{c a}{ }^{* *}=\frac{\mu_{s}^{2} \nu}{\sigma \varsigma 0 \sigma \vartheta} \frac{1}{\mu_{o}}(3)$

$M=\frac{\frac{\mu_{s}}{\mu_{o}}}{\cos \theta}(4)$

$W e=\frac{\rho v^{2} l}{\sigma}(5)$

$W e^{*}=\frac{\rho v^{2} l}{\sigma \varsigma \circ \sigma \vartheta}(6)$

( $\mu_{\mathrm{s}}$ : viscosity of the solution, $\mu_{\mathrm{o}}$ : viscosity of oil, v: velocity, $\sigma$ : surface tension, $\theta$ : contact angle, $\rho$ : solution density, $l$ : characteristic length, viscosity $\left.\operatorname{ratio} \mu^{s} / \mu^{o}\right)$.

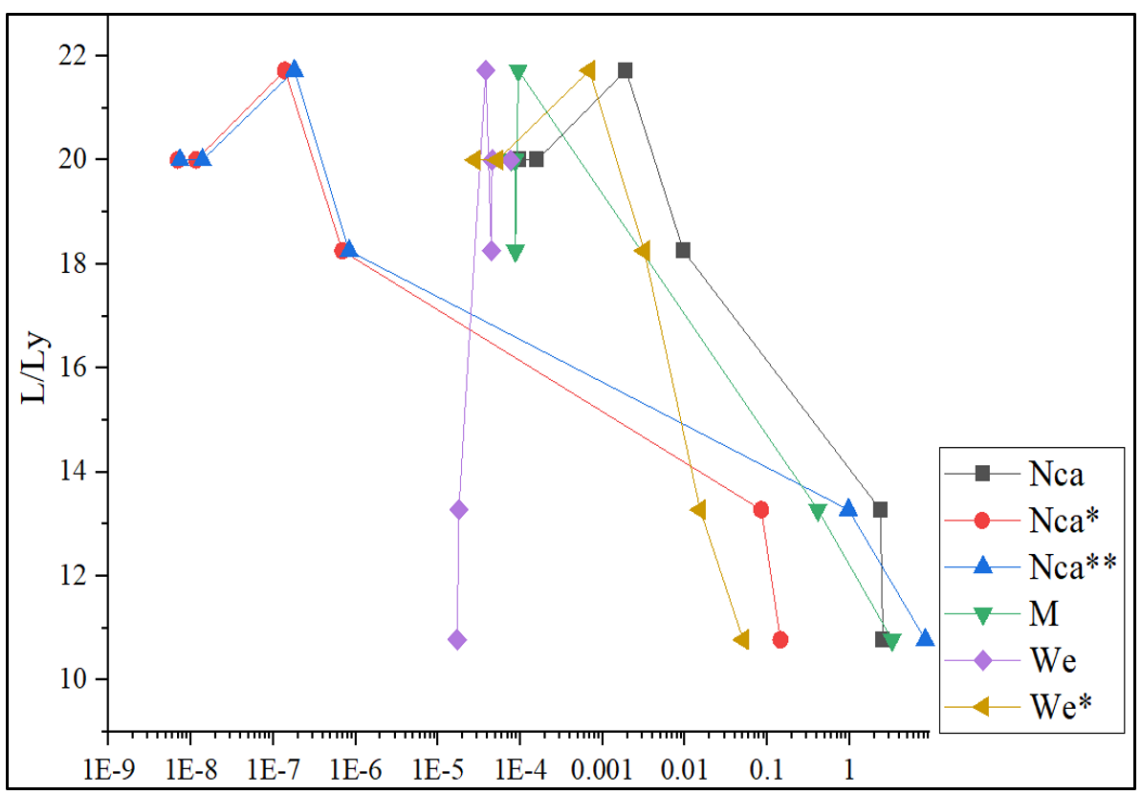




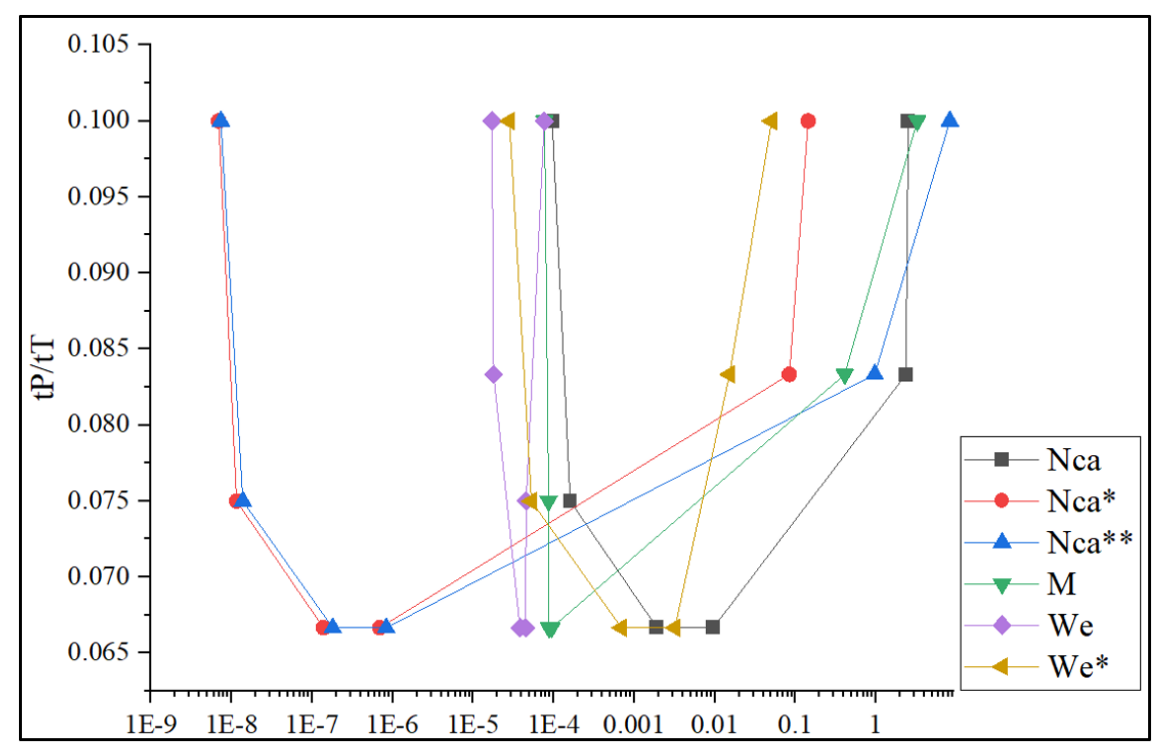

I II

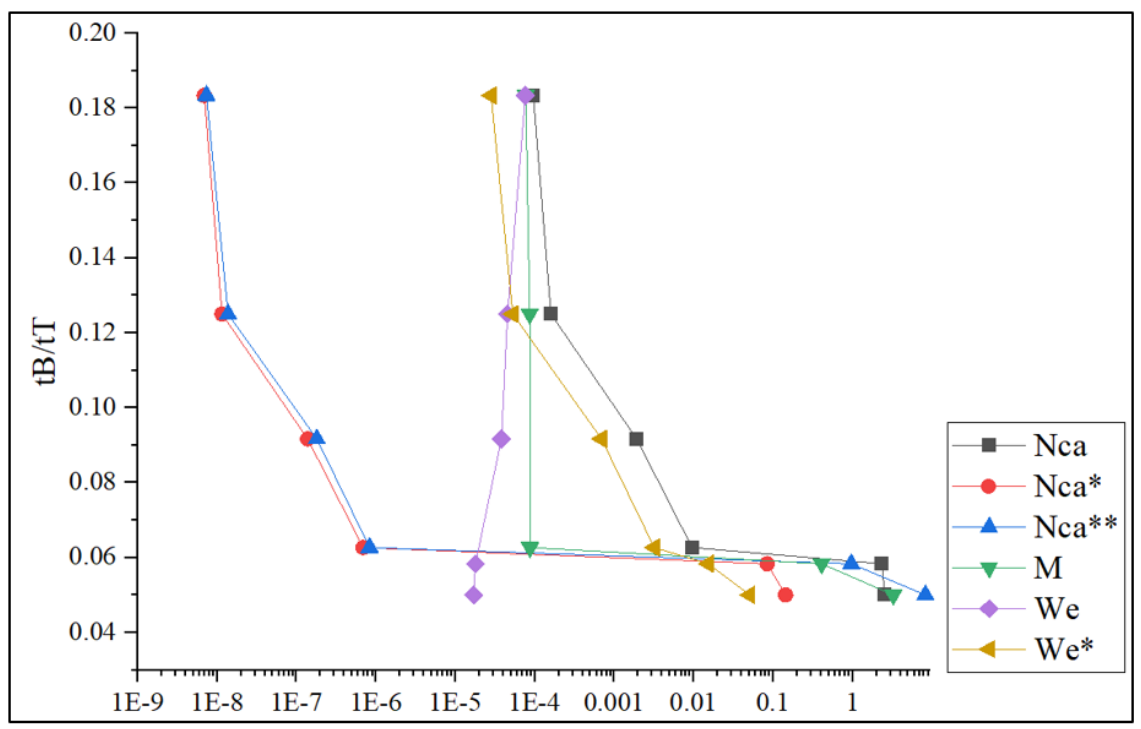




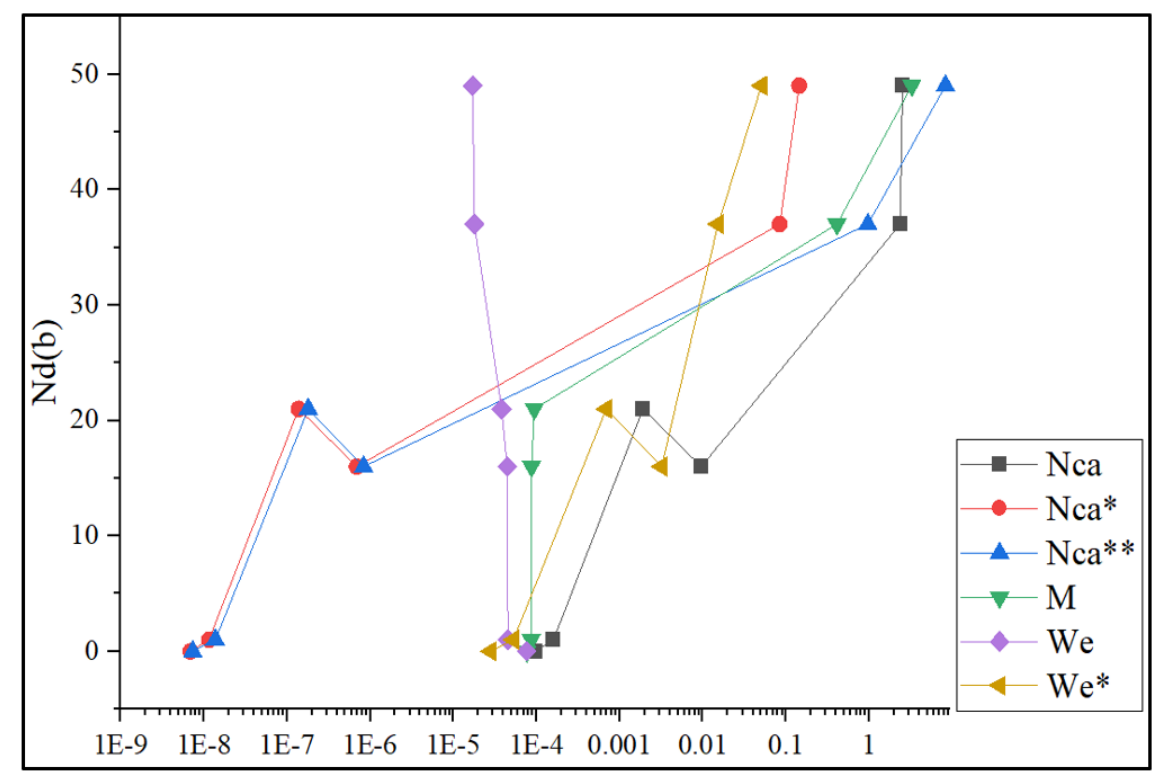

III IV

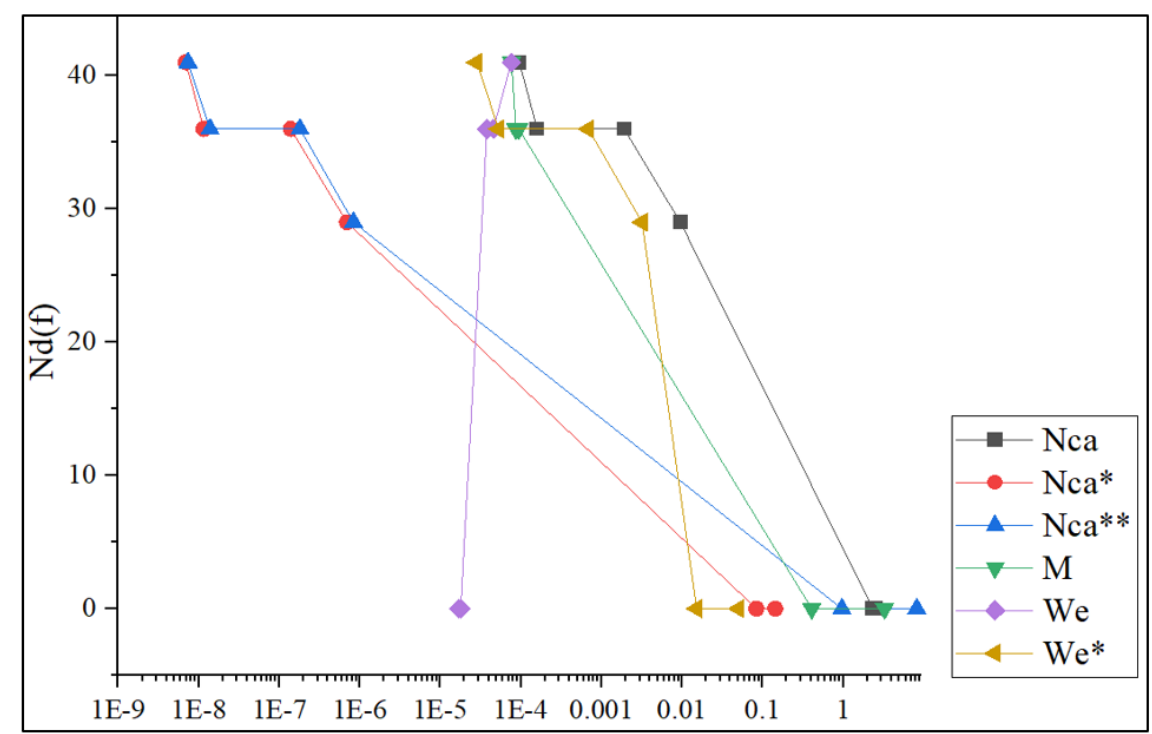

V

Figure 13-Dimensionless numbers vs. descriptive parameters (in log scale) (I) $L / L y$, (II) $t P / t T$, (III) $t B / t T$, (IV) $N d(\mathrm{~b}),(\mathrm{V}) N d(f)$.

Figure 13 demonstrates the functional relationship between the descriptive parameters (I) $L / L y$, (II) $t P / t T$ , (III) $t B / t T$, (IV) $N d(b),(\mathrm{V}) N d(f)$ and dimensionless numbers $\left(N_{c a}{ }^{*}, N_{c a}{ }^{* *}, M, N_{c a}, W e, W e^{*}\right)$.

(I) $L / L y$ vs. dimensionless scaling groups. Overall trends are characterized by a decrease of $L / L y$ following an initial increase as the dimensionless numbers increase. The slow decrease could also be observed in the plots with $N_{c a}{ }^{*}$ and $N_{c a}{ }^{* *}$, while fast decrease could be seen in the case of $M$, and $N_{c a} . L / L y$ plotted with $W e^{*}$ also displayed a relatively fast decrease compared to that of other dimensionless numbers. L/Ly vs. 
We, however, demonstrated a divergent trend from the rest of the plots characterized by a steep increase in $L / L y$ following a sudden dip. Considering that the magnitude of the considered viscosity term for each case is in the ratio of 2:1:0 $\left(N_{c a}{ }^{*}, N_{c a}{ }^{* *}: M, N_{c a}: W e, W e^{*}\right.$, respectively), viscosity force can be attributed to being the leading factor in determining the normalized interfacial length of fingers before their stability loss. With the larger predominance of the viscosity effect, $L / L y$ development was shown to be delayed. Such behavior could be due to the repressed shielding effect as elaborated in the study by Nagatsu et al. (2007).

(II) $t P / t T$ vs. dimensionless scaling groups. The general trends for the dimensionless groups plotted with normalized production port reaching time display an initial decrease followed by a stabilized phase and subsequent increase. The length of the stagnant period, however, was observed to vary and appeared to be shorter in the plots with $W e$ and $M$. Considering that these two dimensionless groups do not share any variables, several factors can be considered to be the cause for the existence of the stagnant period. The chemicals responsible for the stagnant behavior are chemical samples whose contact angle and surface tension are relatively similar in range compared to that of other chemical samples which indicates the sensitivity of $t P / t T$ to the wettability and IFT effect.

(III) $t B / t T$ vs. dimensionless scaling groups. The general decreasing tendency can be observed in the plots except for the case of $t B / t T$ vs. $W e$. In the case of $W e$, with the increase in $W e, t B / t T$ increased due to the exclusive effect of surface tension. However, its impact is immediately minimized when other variables such as wettability and viscosity effect are considered in the rest of the dimensionless numbers. In addition, the viscosity effect is observed to be responsible for the long-stagnant period which exists in the plots with $N_{c a}{ }^{*}, N_{c a}{ }^{* *}$, and $M$, following an initial steep decrease. The combination of surface tension and contact angle effect seems to be the responsible factor for the steep slope of decrease (with the relatively shorter period of stagnation) observed in the case of $W e^{*}$ and $N_{c a}$.

(IV) $N d(b)$ vs. dimensionless scaling groups. The increasing tendency could be observed in all cases except for We which demonstrated that the number of droplets before the "finger break" decreases with the decrease in surface tension (regardless of the viscosity which has the function of stabilizing the hydrodynamic instability). This is due to the hydrodynamic stability of fingers associated with low surface tension. However, considering that the opposite trend is observed to hold for the rest of the cases, it can be concluded that while low IFT can maintain hydrostatic stability of the fingers, it plays a minimal role in determining $N d(b)$ when other forces such as viscosity and wettability effect are considered.

(V) $N d(f)$ vs. dimensionless scaling groups. The overall decreasing tendency could be observed in the plots except in the case of $W e$ which again indicates that lower surface tension is associated with an increase in the number of droplets after the finger break (hydrodynamic stability loss). This is an interesting phenomenon which indicates loss of hydrodynamic stability leads to the generation of a number of droplets from the finger pinch off for the low IFT cases (Figure 14 ). There are two well-established coarsening mechanisms for emulsion: coalescence and Ostwald ripening. Coalescence occurs due to the fusion of droplets while Ostwald ripening is caused by the molecular exchange through the continuous phase. Visual data analysis of the samples clearly demonstrated that the cause of emulsion coarsening in the partial-miscibility fingering like state (brought on by hydrostatic instability) is Ostwald ripening, rather than coalescence.

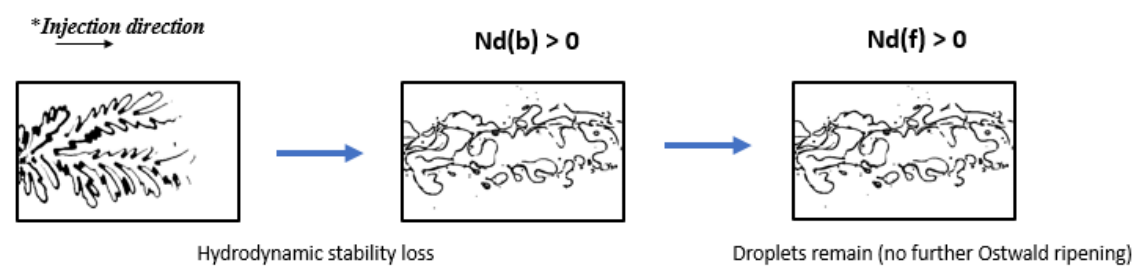

Figure 14-Surfactant induced fingering stability.

Ostwald ripening also occurred in the high viscosity case (polymer-induced) where surfactant- induced fingers 
were consumed by the larger-sized polymer fingers as the surfactant induced fingers lost their hydrodynamic stability (Figure 15 ). Polymer-induced hydrodynamic stability then dominated the overall finger morphology rendering the number of droplets zero. Polymer-induced fingers are also observed to follow the original finger patterns of the surfactant-induced fingers however, as they consumed and developed into new morphologies. In our previous study (Lee et al. 2020), polymeric nanofluid (cellulose nanocrystals) was also observed to demonstrate a similar behavior and such effect became more pronounced as the viscosity of the solution increased over time (increase in hydrostatic stability).

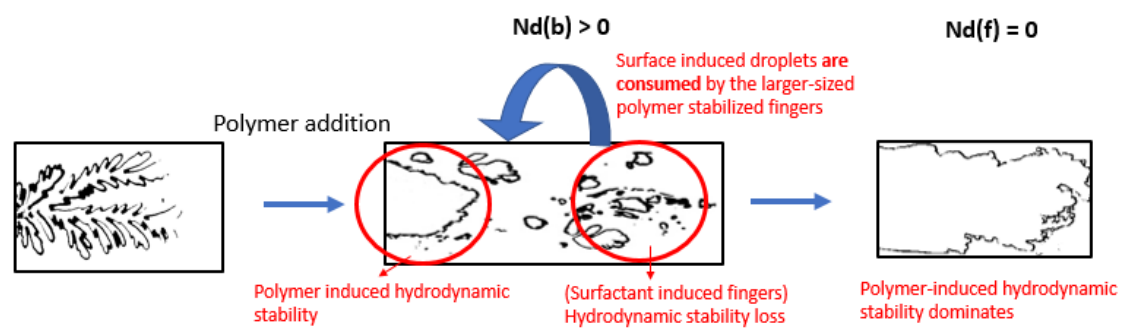

Figure 15- Surfactant and polymer-induced fingering stability (injection direction available in Figure 14).

As a continuation of the analysis above, $N d(f)$ is the normalized number of droplets at the final stage of the experiment. And as previously elaborated, the droplet number is a primary indicator of viscous fingering hydrostatic instability. Figure $\mathbf{1 6}$ displays the combination of visual and quantitative analysis results for each dimensionless number case. $N_{c a}{ }^{*}$ plot demonstrates that with an increase in the viscous forces, after reaching a specific point $\left(>10^{-9}\right), N d(f)$ decreases consistently while fingers become more stable. $N_{c a}$ plot follows a similar trend - however, stable finger morphology driven by viscous forces appeared in the lower magnitude ranges while stable finger morphology (higher number of tip splits) dominated by surface tension came after. Overall, droplet formation was observed to be in "low to intermediate ranges" of the dimensionless numbers $\left(N_{c a}: 10^{-4} \sim 10^{-2}, N_{c a}{ }^{*}: 10^{-9} \sim 10^{-6}, N_{c a}{ }^{* *}:<10^{-7}, W e: 10^{-5} 10^{-3}, W e^{*}:<10^{-2}, M: 10^{-4}\right)$. Note that each finger morphology and its corresponding dimensionless number ranges were assigned to a unique color band for better visualization of the graphs.

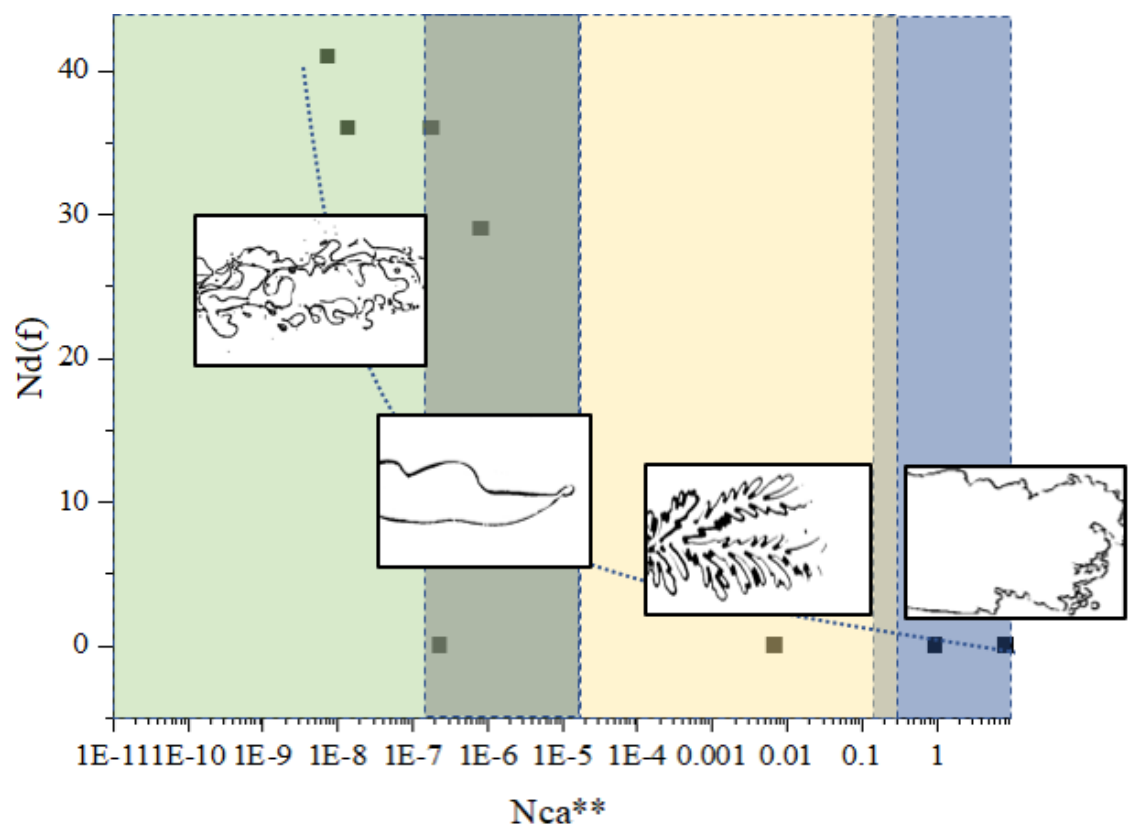



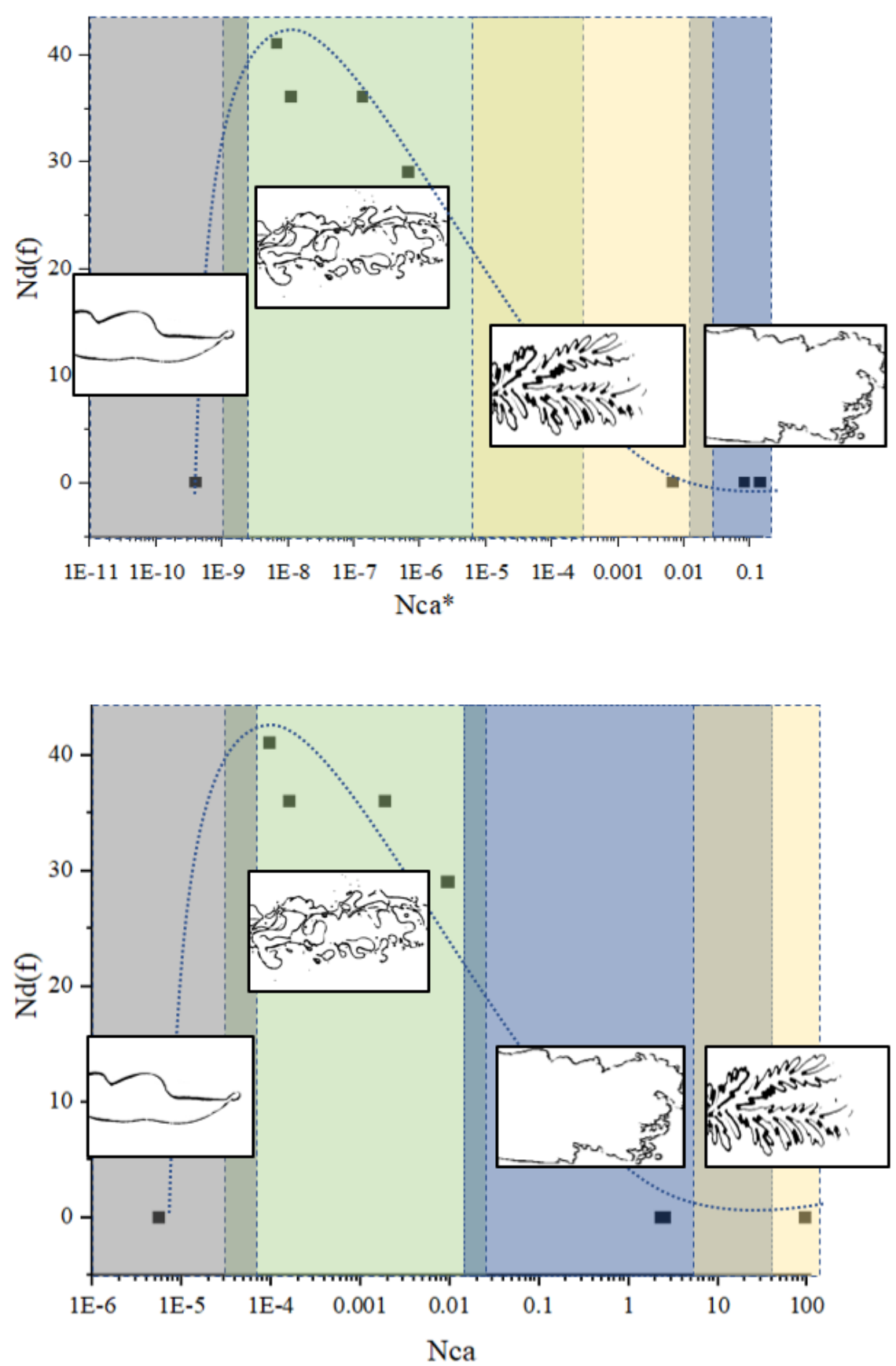

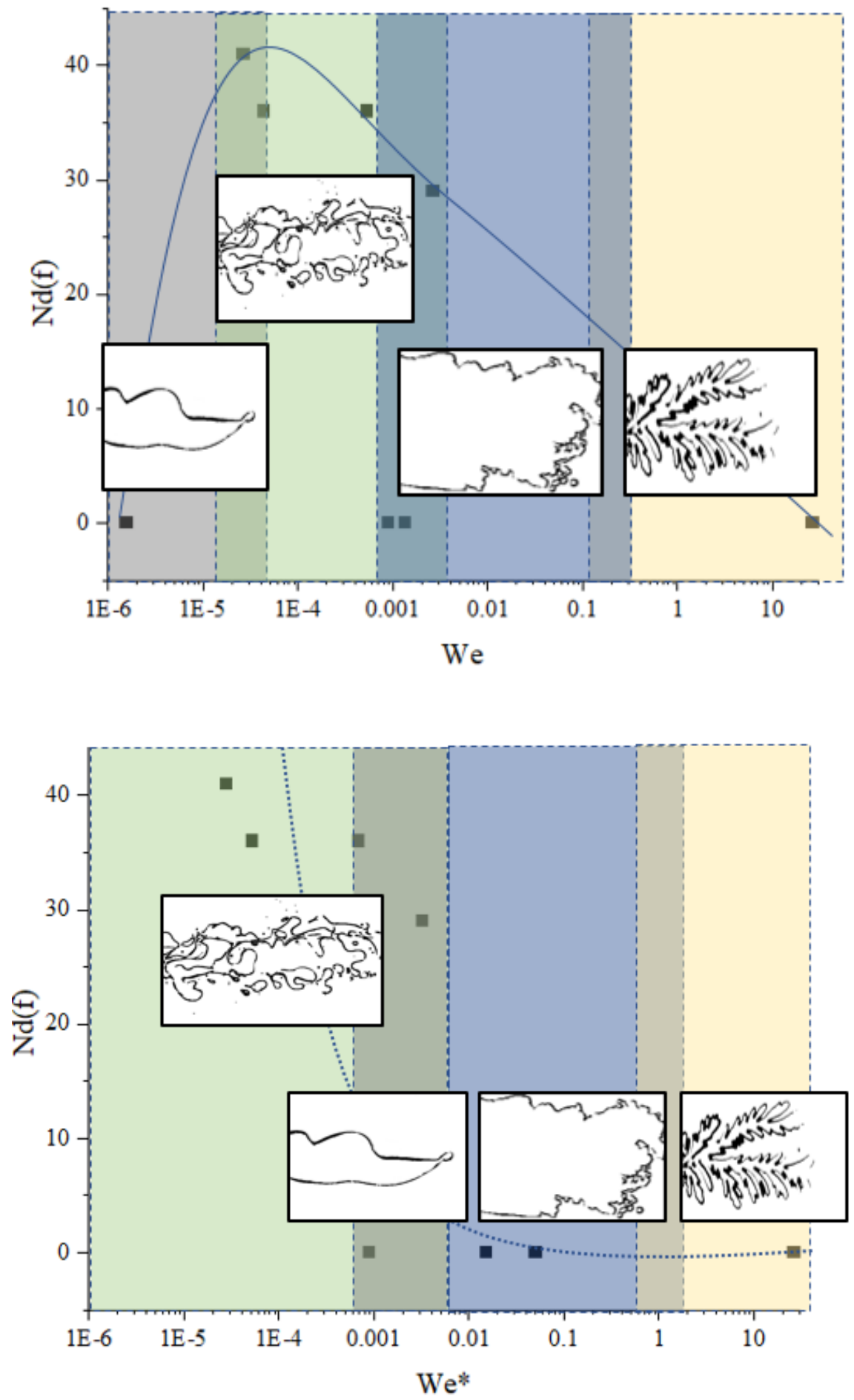


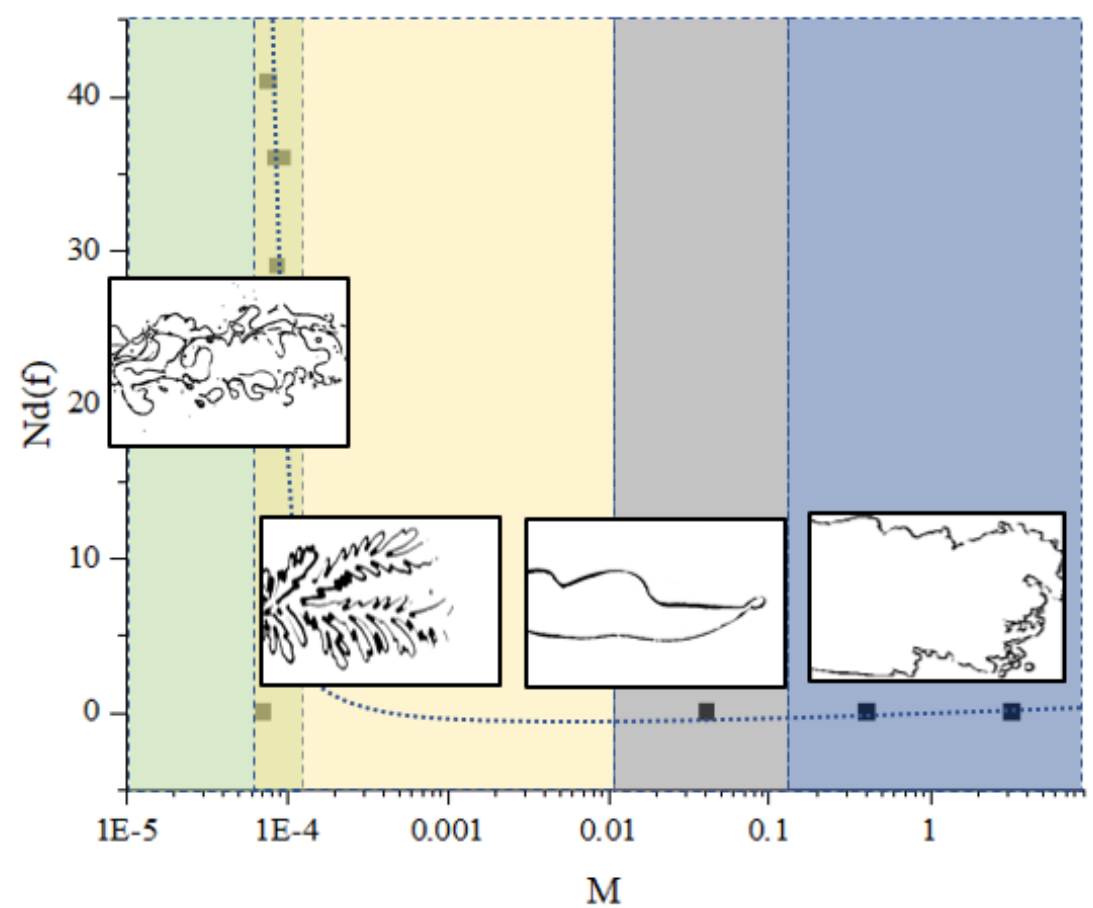

Figure 16-Description of emulsion development and $\mathrm{Nd}(\mathrm{f})$.

Fu et al. (2016) stated that the pinch-off from the viscous fingering instability results in small droplets ending up being consumed by larger droplets through diffusive mass transfer via Ostwald ripening; however, if the diffusive mass transfer rate cannot compete with the rate of smaller droplet generation, it may lead to excess dissolved concentration and a supersaturated liquid phase. And with disturbance, droplets can be generated. However, in this study, despite the constant injection of the solution ("disturbance"), it could be seen after a certain period, the droplets remained - not to be further consumed by the surrounding largersized droplets (and co-existing fingers) which implies that surface energy was sufficiently low, and further energy minimization was not required. The cause of this behavior requires further investigation. However, it can be speculated that the IFT between the phases in the overall Hele-Shaw cell had reached the CMC (Critical Micelle Concentration) value and therefore, there was not an active chemical interaction between the liquid phases which could cause a disturbance. In which case, droplet development (or lack thereof) during an emulsion flow in a Hele-Shaw model can be employed as an indicator to predict the "in-situ" CMC of injected surfactants.

Provided in Figure $\mathbf{1 7}$ are the micrographs and particle size distribution histograms of the oil-in-water macroemulsion samples produced from the injection experiments. Particle size distribution histogram tended to have the large tail towards the left and generally followed a lognormal distribution pattern. It can be seen that larger sized droplets are associated with the more stable emulsion groups while the smaller sized droplets are associated with the less stable emulsion groups (associated with high $N f(d)$ ). The stability of emulsions for the upper cases in Figure 17a was induced by the polymer. Hydrophilic polymers are effective in preventing coalescence by increasing the bulk phase viscosity, reducing the kinetic energy of droplet particles, and controlling the collisions accordingly (Sadtler et al. 2002). The cause for the coarser nature of emulsions stabilized by polymeric solutions is not well-studied. However, it should be noted that oil displacement was more effective in the stable emulsion case, hence the higher oil content (and larger droplet size) observed in the micrographs. The micrographs available in Figure 17b were captured after adding an extra mechanical force via agitation, and before the agitation, produced samples easily separated into two phases due to the emulsion kinetic instability. When adding the external force to the upper glass samples however, there was 
no significant change in the droplet sizes or the droplet size distribution.
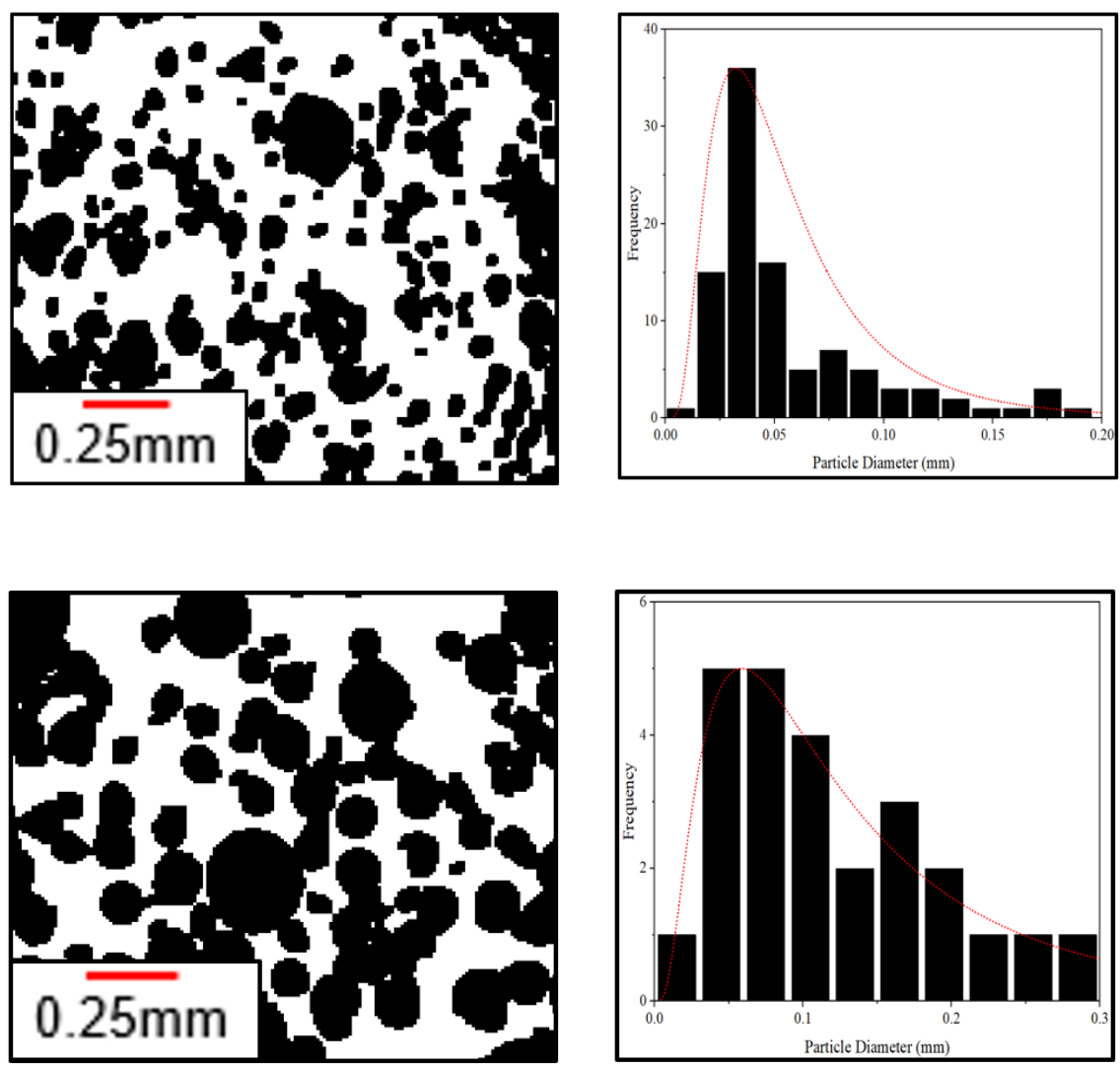

(a)
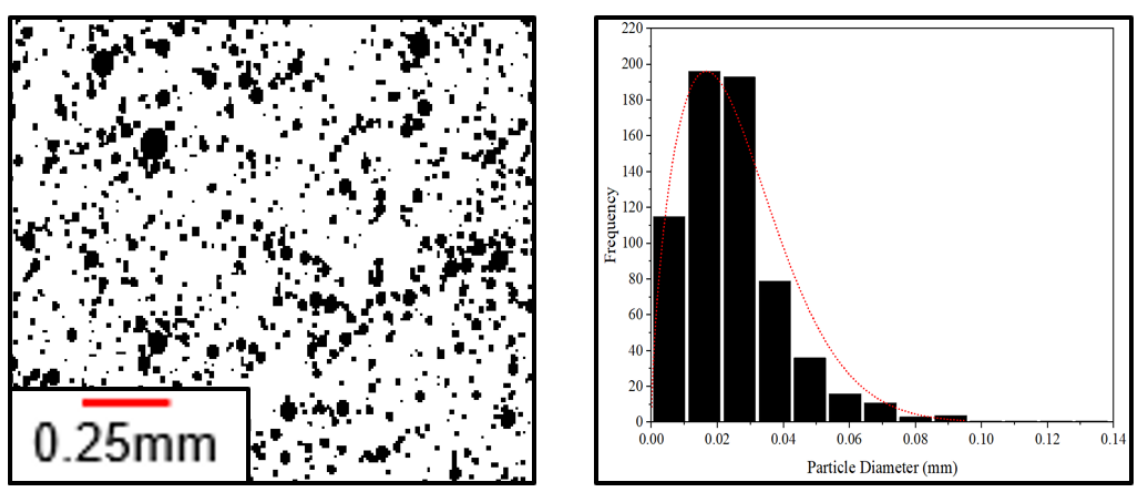

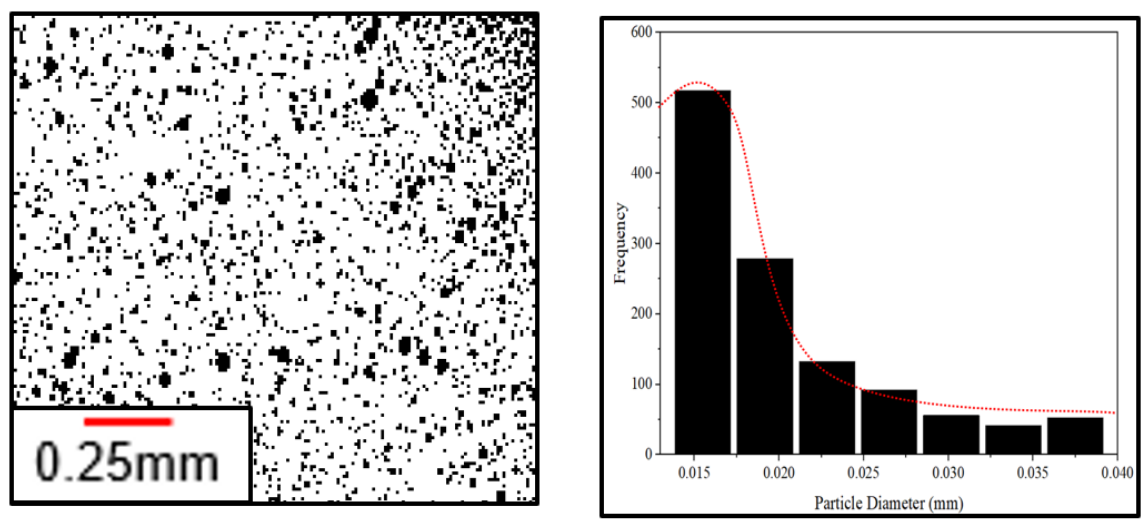

(b)

Figure 17-Micrographs and particle size distribution (a) stable emulsion (b) unstable emulsion after the agitation.

\section{Discussion}

Despite the availability of a large quantity of literature on viscous fingering (immiscible and miscible), few have addressed the viscous finger types that involve chemical interaction. And emulsion flow-especially that of heavy oil has been mostly neglected. In this paper, we attempt to address this missing gap by approaching emulsion flow through fractal analysis and quantification analysis using various dimensionless numbers. Based on this analysis, we have provided the phase map to describe the overall relationship with hydrodynamic stability, surface tension, and viscosity using the original experimental images. The fractal dimension $\left(D_{\mathrm{B}}\right)$ corresponding to each image has also been provided (Figure 18). It can be inferred that tip-splitting like behavior is associated with miscibility at low viscosity conditions (and higher fractal dimensions). In this emulsion flow study, with the hydrodynamic stability loss, droplet formation begins until it eventually reaches the steady-state of energy and droplets maintain their shape. The mechanism for the temporary or permanent halt in the coarsening process in the case of surfactant-induced fingering requires further investigation - -although CMC may be suspected to be the cause. In the case of fingering induced by surfactant and polymer, viscosity plays a significant role in maintaining the hydrodynamic stability, and surfactant-induced fingers develop before the development of the polymer-induced fingers (which also have higher hydrodynamic stability) - which eventually results in the consumption of surfactant-induced fingers by polymer-induced fingers via Ostwald ripening. Polymer-induced fingers are characterized by a smooth frontal mobility line along with a high level of hydrodynamic stability subsequently rendering droplet generation non-existent. 


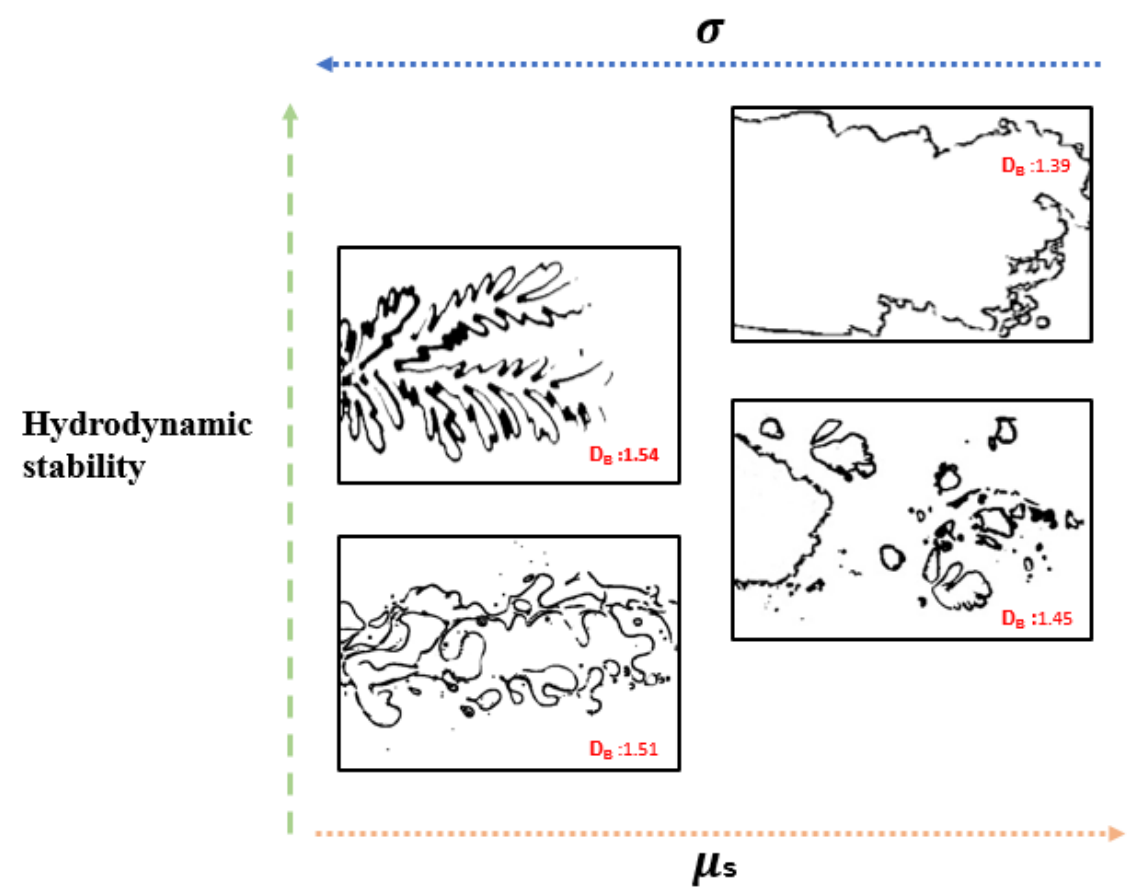

Figure 18-Hydrodynamic stability, viscosity, surface tension and fractal dimension $\left(\mathrm{D}_{\mathrm{B}}\right)$.

As expected, higher fractal dimensions were associated with fingers with the higher tip splitting capacity while lower fractal dimensions were associated with fingers with the smooth frontal line. Hydrodynamic stability was not observed to have any direct correlation with the fractal dimensions due to the constant fingering deformation.

\section{Conclusions}

1. Droplet formation observed in the partially-miscible Hele-Shaw studies is associated with emulsion instability: produced emulsions from the droplet morphologies require an external mechanical force to maintain their stability.

2. Emulsion stability is predominantly dependent on the viscosity force. With the increase in the strength of the viscous force, the number of droplets decreases. This effect is especially pronounced at the later stage of the experiment for cases that contain both the surface-active agent and the viscosifying agent. Surfactantinduced fingers are consumed by large and highly-stable polymer-induced fingers via the Ostwald ripening process.

3. Low IFT is associated with the droplet generation after the initiation of the finger break. The opposite is observed to be true (less droplet generation) before the finger stability loss (at earlier stages of the experiment) which implies that low IFT is a leading factor in hydrodynamic stability at the initial stage. However, upon the stability loss, droplets are generated and such droplet generation continues until it begins to plateau.

4. Finger development before and after the stability loss follows separate self-similarity patterns and such divergence in patterns can be noted in saturation profiles plotted with the time variable.

5. Fractal dimensions have a proportional relationship with surface tension and an inversely proportional relationship with viscosity.

6. We presented two types of "self-similar" behaviors per the categorization based on the saturation profiles. 
However, certain complex colloidal samples may display multiple self-similar fingering behaviors based on the chemical component, mechanism, and subsequently induced hydrodynamic stability (or instability).

7. For the future study (in oil recovery), the impact of droplet generation behavior on heavy oil recovery in the porous media can be investigated. Pore size distribution can be predicted to be the most significant parameter on the droplet retainment behavior which can improve the overall oil recovery via entrapment (mobility control), or reduce it by decreasing the relative permeability to oil.

\section{Acknowledgments}

This research was conducted under the third author's (TB) NSERC Industrial Research Chair in Unconventional Oil Recovery (industrial partners are Petroleum Development Oman, , Husky Energy, Saudi Aramco, CNRL, Suncor, and BASF) and an NSERC Discovery Grant (No: RES0011227). We gratefully acknowledge these supports.

\section{References}

Abramoff, M., Magalhaes, P., and Ram, S. 2004. Image Processing with ImageJ.Biophotonics International 11 (7): $36-42$.

Al-Bahlani, A. and Babadagli, T. 2012. Visual analysis of diffusion process during oil recovery using a Hele-Shaw model with hydrocarbon solvents and thermal methods. Chemical Engineering Journal 181-182 : 557-569. https://doi.org/10.1016/j.cej.2011.11.087.

Arnéodo, A., Couder, Y., Grasseau, G. et al. 1989. Uncovering the analytical Saffman-Taylor finger in unstable viscous fingering and diffusion-limited aggregation. Physical Review Letters ,63 (9), 984-987. https://doi.org/10.1103/physrevlett.63.984.

Bischofberger, I., Ramachandran, R., and Nagel, S. 2014. Fingering versus stability in the limit of zero interfacial tension. Nature Communications 5 (1). https://doi.org/10.1038/ncomms6265.

Bonn, D., Kellay, H., Ben Amar, M. et al. 1995. Viscous finger widening with surfactants and polymers. Phys. Rev. Lett . 75 : 2132-2135. https://doi.org/10.1103/PhysRevLett.75.2132.

Bruns, F., and Babadagli, T. 2020. Heavy-oil recovery improvement by additives to steam injection: Identifying underlying mechanisms and chemical selection through visual experiments. Journal of Petroleum Science and Engineering 188 : 106897. https://doi.org/10.1016/j.petrol.2019.106897.

Chen, J. 1989. Growth of radial viscous fingers in a Hele-Shaw cell.Journal of Fluid Mechanics 201 (1): 223. https://doi.org/10.1017/s0022112089000911.

Chui, J., de Anna, P., and Juanes, R. 2015. Interface evolution during radial miscible viscous fingering. Physical Review E 92( 4). https://doi.org/10.1103/physreve.92.041003.

Chuoke, R., Van Meurs, P., and Van der Poel, C. 1959. The instability of slow, immiscible, viscous liquid-liquid displacement in permeable media. Transactions of the AIME 216: 188-194. SPE-1141-G. https://doi.org/10.2118/1141-G.

De Wit, A. 2001. Fingering of Chemical Fronts in Porous Media.Physical Review Letters 87 (5). https://doi.org/10.1103/physrevlett.87.054502.

Fang, F. and Babadagli, T. 2016a. 3-D Visualization of Diffusive and Convective Solvent

Transport Processes in Oil Saturated Porous Media Using Laser Technology. J. of Visualization 19(4): 615-629. https://doi.org/10.1007/s12650-016-0350-1.

Fang, F. and Babadagli, T. 2016b. Three Dimensional Visualization of Solvent Chamber Growth 
in Solvent Injection Processes: An Experimental Approach. J. Petr. Sci. and Eng. 142: 46-67. https://doi.org/10.1016/j.petrol.2016.02.001.

Fang, F. and Babadagli, T. 2016c. Dynamics of Diffusive and Convective Transport in Porous

Media: A Fractal Analysis of 3-D Images Obtained by Laser Technology. Chaos, Solitons 83 Fractals 95: 1-13. https://doi.org/10.1016/j.chaos.2016.11.015.

Ferer, M., Sams, W., Geisbrecht, R. et al. 1993. The Fractal Nature of Viscous Fingering:

Saturation Profiles and Fractional Flows From Modeling of Miscible, Two-Component Flows in TwoDimensional Pore Level Models. SPE Symposium on Reservoir Simulation, New Orleans, Louisiana, 28 February-3 March. SPE-25270-MS. https://doi.org/10.2118/25270-ms.

Ferer, M., Ji, C., Bromhal, G. et al. 2004. Crossover from capillary fingering to viscous fingering for immiscible unstable flow: Experiment and modeling. Physical Review E $\mathbf{7 0}$ (1). https://doi.org/10.1103/physreve.70.016303.

Fernandez, J. and Homsy, G. 2003. Viscous fingering with chemical reaction: effect of in-situ production of surfactants. Journal of Fluid Mechanics480 : 267-281. https://doi.org/10.1017/s0022112002003683.

Fu, X., Cueto-Felgueroso, L., and Juanes, R. 2016. Thermodynamic coarsening arrested by viscous fingering in partially miscible binary mixtures. Physical Review E 94 (3). https://doi.org/10.1103/physreve.94.033111.

Fu, X., Cueto-Felgueroso, L., and Juanes, R. 2017. Viscous fingering with partially miscible fluids. Physical Review Fluids 2 (10). https://doi.org/10.1103/physrevfluids.2.104001.

Gharbi, R., Qasem, F., and Peters, E. 2001. A relationship between the fractal dimension and scaling groups of unstable miscible displacements.Experiments in Fluids $\mathbf{3 1}$ (4), 357-366. https://doi.org/10.1007/s003480100278.

Hamida, T. and Babadagli, T. 2007. Fluid-fluid interaction during miscible and immiscible displacement under ultrasonic waves. The European Physical Journal B 60 (4), 447-462. https://doi.org/10.1140/epjb/e200800005-5.

Jackson, S., Power, H., Giddings, D. et al. 2017. The stability of immiscible viscous fingering in Hele-Shaw cells with spatially varying permeability. Computer Methods in Applied Mechanics and Engineering 320 : 606-632. https://doi.org/10.1016/j.cma.2017.03.030.

Jha, B., Cueto-Felgueroso, L., and Juanes, R. 2013. Synergetic Fluid Mixing from Viscous Fingering and Alternating Injection. Physical Review Letters 111 (14). https://doi.org/10.1103/physrevlett.111.144501.

Kawaguchi, M., Yamazaki, S., Yonekura, K. et al. 2004. Viscous fingering instabilities in an oil in water emulsion. Physics of Fluids16 (6): 1908-1914. https://doi.org/10.1063/1.1709543.

Lee, J., Babadagli, T., and Giesbrecht, R. 2019. Impact of Divalent Ions on Heavy Oil Recovery by in situ Emulsification. Journal of Surfactants and Detergents 22 (6): 1371-1385. https://doi.org/10.1002/jsde.12345.

Lee, J., Huang, J., and Babadagli, T. 2020. Dynamics of emulsion generation and stability during heavy oil displacement with chemicals and nanoparticles: Qualitative analysis using visual 2D data.Fuel 270 : 117502. https://doi.org/10.1016/j.fuel.2020.117502.

Mandelbrot, B. 1983. The Fractal Geometry of Nature (pp. 42-43). New York, W.H. Freeman and company.

Maxworthy, T. 1986. Bubble formation, motion and interaction in a Hele-Shaw cell. Journal of Fluid Mechanics 173 : 95-114. https://doi.org/10.1017/s002211208600109x.

May, S. and Maher, J. 1989. Fractal dimension of radial fingering patterns. Phys. Rev. A40 (3): 1723-1726. https://doi.org/10.1103/PhysRevA.40.1723. 
Naderi, K., and Babadagli, T. 2011. Visual analysis of immiscible displacement processes in porous media under ultrasound effect. Physical Review E 83 (5). https://doi.org/10.1103/physreve.83.056323.

Nagatsu, Y., Matsuda, K., Kato, Y. et al. 2007. Experimental study on miscible viscous fingering involving viscosity changes induced by variations in chemical species concentrations due to chemical reactions. Journal of Fluid Mechanics 571 : 475-493. https://doi.org/10.1017/s0022112006003636.

Nittmann, J., Daccord, G., and Stanley, H. 1985. Fractal growth viscous fingers: quantitative characterization of a fluid instability phenomenon. Nature 314 (6007): 141-144. https://doi.org/10.1038/314141a0.

Sadtler, V. M., Imbert, P., and Dellacherie, E. 2002. Ostwald Ripening of Oil-in-Water Emulsions Stabilized by Phenoxy-Substituted Dextrans. Journal of Colloid and Interface Science 254 (2): 355-361. https://doi.org/10.1006/jcis.2002.8624.

Saffman, P. and Taylor, G. 1958. The penetration of a fluid into a porous medium or Hele-Shaw cell containing a more viscous liquid. Proceedings of the Royal Society of London. Series A. Mathematical and Physical Sciences 245 (1242): 312-329. https://doi.org/10.1098/rspa.1958.0085.

Sastry, M., Gole, A., Banpurkar, A. et al. 2001. Variation in viscous fingering pattern morphology due to surfactant-mediated interfacial recognition events. Current Sci . 81 (2): 191-193.

Settari, A., Price, H., and Dupont, T. 1977. Development and Application of Variational Methods for Simulation of Miscible Displacement in Porous Media. Society of Petroleum Engineers Journal 17 (3): 228-246. https://doi.org/10.2118/5721-pa.

Sharma, J., Inwood, S., and Kovscek, A. 2012. Experiments and Analysis of Multiscale Viscous Fingering During Forced Imbibition. SPE Journal 17 (4): 1142-1159. https://doi.org/10.2118/143946-pa.

Suzuki, R., Nagatsu, Y., Mishra, M. et al. 2016. Experimental study on viscous fingering with partial miscible fluids. APS Physics. Abstract ID: BAPS.2016.DFD.D38.7.

Tan, C. and Homsy, G. 1992. Viscous fingering with permeability heterogeneity. Physics of Fluids A: Fluid Dynamics 4 (6): 1099-1101. https://doi.org/10.1063/1.858227.

Wang, Y., Liu, H., Zhang, Q. et al. 2020. Pore-scale experimental study on EOR mechanisms of combining thermal and chemical flooding in heavy oil reservoirs. Journal of Petroleum Science and Engineering185 : 106649. https://doi.org/10.1016/j.petrol.2019.106649.

Yortsos, Y. and Zeybek, M. 1988. Dispersion driven instability in miscible displacement in porous media. Physics of Fluids 31 (12): 3511. https://doi.org/10.1063/1.866918. 